Issued by Sandia National Laboratories, operated for the United States Department of Energy by Sandia Corporation.

NOTICE: This report was prepared as an account of work sponsored by an agency of the United States Government. Neither the United States Government, nor any agency thereof, nor any of their employees, nor any of their contractors, subcontractors, or their employees, make any warranty, express or implied, or assume any legal liability or responsibility for the accuracy, completeness, or usefulness of any information, apparatus, product, or process disclosed, or represent that its use would not infringe privately owned rights. Reference herein to any specific commercial product, process, or service by trade name, trademark, manufacturer, or otherwise, does not necessarily constitute or imply its endorsement, recommendation, or favoring by the United States Government, any agency thereof, or any of their contractors or subcontractors. The views and opinions expressed herein do not necessarily state or reflect those of the United States Government, any agency thereof, or any of their contractors.

Printed in the United States of America. This report has been reproduced directly from the best available copy.

Available to DOE and DOE contractors from Office of Scientific and Technical Information P.O. Box 62

Oak Ridge, TN 37831

Prices available from (703) 605-6000

Web site: http://www.ntis.gov/ordering.htm

Available to the public from

National Technical Information Service

U.S. Department of Commerce

5285 Port Royal Rd

Springfield, VA 22161

NTIS price codes

Printed copy: A03

Microfiche copy: A01

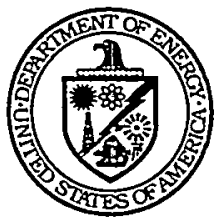




\section{DISCLAIMER}

Portions of this document may be illegible in electronic image products. Images are produced from the best available original document. 
SAND99-8588

Unlimited Release

Printed July 1999

\title{
Using Spatial Gradients to Model Localization Phenomena
}

\author{
Douglas J. Bammann \\ Solid and Material Mechanics Department \\ Daniel Mosher \\ Experimental Mechanics Department \\ Darcy A. Hughes and Neville R. Moody \\ Materials Reliability Department \\ Sandia National Laboratories \\ P.O. Box 969 \\ Livermore, CA 94551-0969 \\ Paul R. Dawson \\ Cornell University \\ Ithaca, NY
}

\begin{abstract}
We present the final report on a Laboratory-Directed Research and Development project, Using Spatial Gradients to Model Localization Phenomena, performed during the fiscal years 1996 through1998. The project focused on including spatial gradients in the temporal evolution equations of the state variables that describe hardening in metal plasticity models. The motivation was to investigate the numerical aspects associated with post-bifurcation mesh dependent finite element solutions in problems involving damage or crack propagation as well as problems in which strain localizations occur. The addition of the spatial gradients introduces a mathematical length scale that eliminates the mesh dependency of the solution. In addition, new experimental techniques were developed to identify the physical mechanism associated with the numerical length scale.
\end{abstract}


This page intentionally left blank 


\section{Contents}

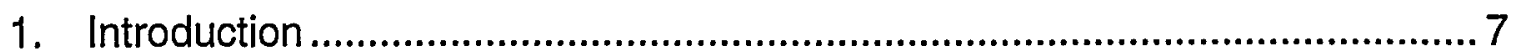

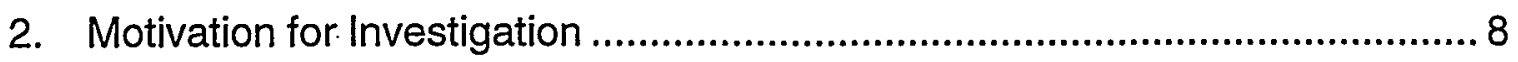

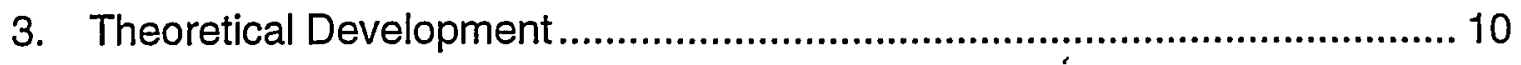

4. Numerical Implementations ..................................................................... 13

4.1 Hardening ....................................................................................... 13

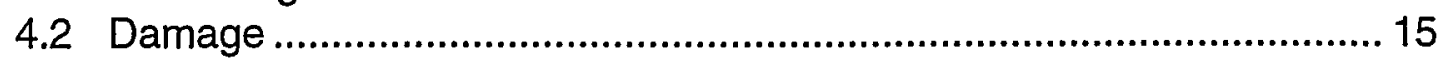

5. Applications ....................................................................................... 17

5.1 Hardening Gradients and Stability .................................................... 17

5.1.1 Overview............................................................................ 17

5.1.2 Variable Threshold Rod Analyses.............................................. 17

5.1.3 Plate with Array of Holes......................................................... 19

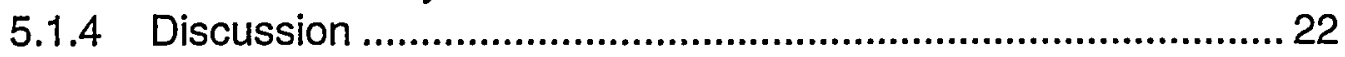

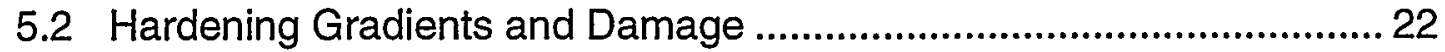

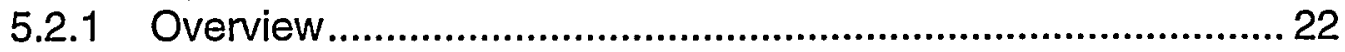

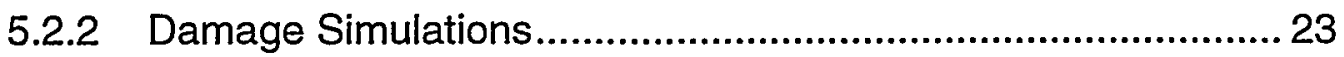

5.2.3 Discussion ………………………………………………......26

5.3 Hardening Gradients and Hardness Profiles.......................................... 26

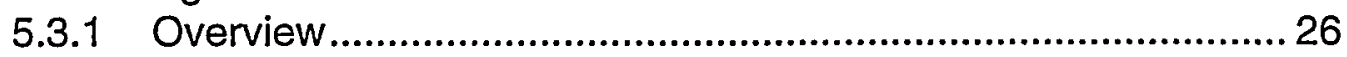

5.3.2 Torsion of Fine Wires...............................................................2 26

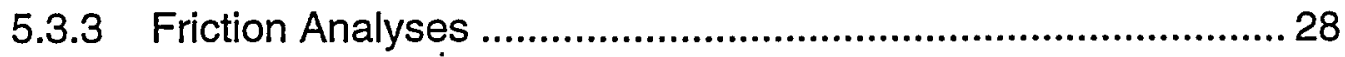

6. Length Scale Parameter Experiments ...................................................... 31

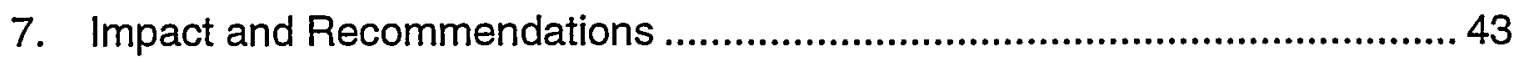

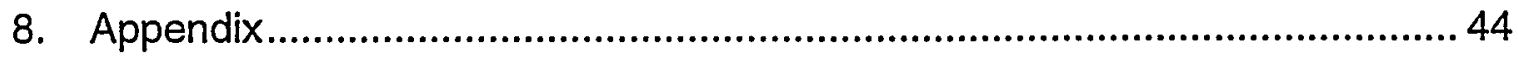


This page intentionally left blank 


\section{Using Spatial Gradients to Model Localization Phenomena}

\section{Introduction}

There has been increased activity in the development and implementation into finite element codes of generalized continua models in recent years. The reasons for these models can loosely be divided into three groups - to overcome numerical difficulties in the post bifurcation regime, to try to imbed more of the physics from smaller length scales into the continuum, and to solve small lengthscale problems which can not accurately be solved with local continuum models. The numerical difficulties arise as more detailed information about both deformation and stress state is demanded in the post failure initiation and post strain localization regimes. The implicit finite element method is a numerical method developed to solve an elliptic system of differential equations describing static mechanical equilibrium. However, once the predicted material response in an element begins to exhibit strain softening (decreasing stress with increasing strain-which we will accept as a loose definition of bifurcation in this discussion), the system of differential equations becomes hyperbolic, while the boundary conditions are prescribed for an elliptic system. A similar situation exists for dynamic shock wave codes where the initially hyperbolic system of differential equations loses hyperbolicity and becomes elliptic at bifurcation. One method of overcoming this difficulty is to introduce non-local or gradient terms in either the evolution of the hardening or the yield surface of the materials.

A second motivation for the introduction of gradient or non-local models stems from an attempt to capture more of the underlying physics of the material, while still utilizing continuum models. A fairly complete review of this type of modeling has been given recently by Aifantis ${ }^{1}$ and will not be duplicated here. However, one of the earliest gradient models proposed within the structure of ColemanGurtin continuum thermodynamics was that of Kratochvil and Dillon ${ }^{2}$, who introduce a second order gradient of strain in the yield surface. This model is very similar to the recently proposed model of Fleck et.al. ${ }^{3}$ who proposed a strain gradient model of plasticity in order to describe the torsion of very thin wires. A series of experiments was performed which showed that local continuum theory could not predict that the stress response depended on the diameter of the wire for very small diameters [3]. The gradient model of Fleck et.al. was able to correctly describe this response. Similar effects have recently been discovered in nano-indentation problems ${ }^{4}$.

In the following, we will attempt to extend existing internal variable models of plasticity to incorporate gradient terms in the evolution of the state variables in a consistent thermodynamic approach and investigate the effects of these terms on several types of mechanical boundary value problems. 


\section{Motivation for Investigation}

In the development of continuum plasticity models there is a trend to incorporate more micro-structural features into the model. This can be accomplished by beginning with small length scales models such as atomistic models, and averaging over several length scales to the continuum level. While this approach may eventually result in models which can be implemented into finite element codes and used in the solution of large boundary value problems, neither the appropriate averaging techniques or the size and speed of computers allow this approach to be used in daily analysis of structures. Another approach is start with a continuum model and attempt to incorporate the physics of smaller length scales. This can be accomplished by introducing internal state variables or "hidden" variables that represent the collective behavior of micro-structural quantities such as phases, precipitates, or geometric defects such as dislocations or voids. Within the construct of this theory, temporal evolution equations are proposed for the state variables, usually based upon analytical or numerical micro-mechanical analyses, and the current value of the internal variables then describes the state of the material at any instant. This approach requires consistent kinematics and thermodynamics, as well as accurate and innovative experimental techniques, a sound physical basis and efficient numerical implementation, to provide a useful tool. Many of the current models are based upon the thermodynamic theory of Coleman and Gurtin ${ }^{5}$. Teodosiu ${ }^{6}$, Kratochvil and Dillon ${ }^{7}$, Kelly and Gillis ${ }^{8}$, Perzyna ${ }^{9}$, and more recently Anand, ${ }^{10}$ Bammann et.al. ${ }^{11}$, McDowell ${ }^{12}$, have all proposed models of this type that include the dislocation density as a state variable. This is not a review paper and therefore the above list of authors represents a sample rather than a comprehensive list. In addition, there is a large class of dislocation based models which are similar, but have not used the Coleman and Gurtin thermodynamics. In most large deformation models, the deformation gradient is multiplicatively decomposed into elastic and plastic parts. This decomposition was proposed in the development of the continuum theory of dislocations in 1957 by Bilby ${ }^{13}$, and later independently introduced in 1967 by Lee and Liu ${ }^{14}$ to describe the kinematics associated with a phenomenological plasticity model. Upon loading through the elastic deformation, a natural or stress free configuration is reached. This definition of the natural configuration couples the kinematics with the constitutive response in that the stress is assumed proportional to the elastic deformation. Elastic, plastic and total strain tensors are then defined in terms of the deformation gradients. The total strain in the natural configuration is easily shown to be the sum of the elastic and plastic strain tensors in that configuration. Similar relations are developed for the velocity gradients. 
The free energy with respect to the natural configuration is the assumed to depend upon the elastic strain, the internal variables and temperature. After imposing conditions of symmetry and invariance, restrictions are placed upon the form of the evolution of the state variables utilizing the dissipation inequality. Parameters of the models must be determined from appropriate experimental results; the models can be implemented into finite element codes. The models have been applied in the solution of complex boundary value problems with excellent results. Unfortunately, at bifurcation, such as occurs when strain softening begins in the case of damage or strain localization, the initially elliptic system of differential equations become hyperbolic, and the problem becomes ill posed due the boundary conditions which are no longer consistent with the system of equations. This means that the models are not useful in the solution of problems past the initiation of strain localization or strain softening due to the initiation of damage. The solutions are mesh dependent in that refinement of the finite element mesh results in a change of the solution ${ }^{15}$. In order to extend the range of. validity of internal state variable theories, many efforts have been devoted to adding degrees of freedom to the continuum, thereby introducing a mathematical length scale into the model. Needleman ${ }^{16}$ has introduced a nonlocal integral term in the evolution of the damage variable in the Gurson model to account for the effects of surrounding material on the response of the continuum point. Similar models have been proposed as discussed in the introduction.

These generalized continua models are similar to model previously proposed, not for numerical reasons, but to embed the continuum models with more degrees of freedom in order to capture more of the physics at smaller length scales. A review of these non-local or gradient models is given by Aifantis [1]. We will mention a few of these models that are pertinent to the development of the current work. Kratochvil and Dillon [2] proposed a finite deformation strain gradient theory of plasticity within the framework of Coleman and Gurtin [5]. They assumed that the free energy also depends upon the spatial gradient of the plastic strain and developed the theory as previously described. Holt ${ }^{17}$ developed a dislocation model to describe the formation of subgrains, by including higher order spatial gradients of the dislocation density in his description. Pielcki ${ }^{18}$ included a Second order spatial gradient or diffusive term in the evolution of the dislocation density to attempt to describe the motion of dislocation during fatigue. Nunziato and Cowin ${ }^{19}$ used continuum mixture theory to develop a gradient theory of voids, which related to fatigue of materials in the elastic region. Bammann and Aifantis ${ }^{20}$ used a form of continuum mixture theory to introduce spatial gradients in the evolution of the dislocation density. The continuum was assumed to exist of three states: a perfect lattice, and mobile and immobile dislocation density states. Within this formulation they were able to recover many of the existing models. Walgraf and Aifantis ${ }^{21}$ proposed a dislocation model similar to the equations developed in Bammann and Aifantis, 
but based upon the more physical thermodynamics of Priggogine. This theory was called reaction-diffusion and resulted in a large body of research in this area.

Recently, Fried and Gurtin ${ }^{22}$ have extended the thermodynamic theory of Coleman and Gurtin [5], by requiring the thermodynamic stresses associated with each state variable to satisfy a micro-force balance law. This approach has been very useful in recovering most of the models of phase field theory, with the important distinction of allowing a consistent method to include deformations in the theory. We will use this approach to develop simple gradient models of dislocation evolution and investigate the effects in these terms on macroscopic boundary value problems.

\section{Theoretical Development}

The thermodynamics we employ, directly follow the theory proposed by Fried and Gurtin [22]. Since we are attempting use this theory to introduce gradient effects in the evolution of the dislocation density, we will initially neglect deformation and temperature. This will simplify the analysis and make it easier to understand the concepts of the theory, and the addition of these variables will not change the results we attain. The deformation has been added in a thermodynamic treatment using the dislocation density as a state variable by Grach et.al. ${ }^{23}$ and in a theory using the gradient of plastic strain as a state variable by Gurtin. ${ }^{24}$ Consider a region of the body $R$, with boundary $\partial R$. The dislocation density within the region will be described by the state variable, $\rho$, and the micro-force acting within the region associated with the presence of the dislocations is given by $\pi$. This micro-force is actually the resolved shear stress component of internal stress field acting on a particular slip system. There is also a similar external micro-force, but consistent with neglecting deformations, it will also be neglected. The effects of neighboring regions on the dislocation state in $R$ is introduced through the stress vector $\chi$ which acts across the boundary $\partial R$. These forces are required to be self-equilibrating at this length which is imposed by requiring that they satisfy a micro-force balance given by,

$$
\operatorname{div}(\chi)+\pi=0
$$

For this simplified theory, the dissipation inequality requires that the rate of change of the free energy is less than or equal to the rate of work done on the body:

$$
\dot{\psi} \leq \chi \cdot \nabla \rho+\pi \dot{\rho}
$$


We assume that the free energy and micro-forces depend upon the dislocation density and the gradient and time rate of change of dislocation density. Then,

$$
\begin{aligned}
& \psi=\hat{\psi}(\rho, \nabla \rho, \dot{\rho}) \\
& \chi=\hat{\chi}(\rho, \nabla \rho, \dot{\rho}) . \\
& \pi=\hat{\pi}(\rho, \nabla \rho, \dot{\rho})
\end{aligned}
$$

Now substituting equation (0.3) into equation (0.2) and rearranging,

$$
\left(\chi-\frac{\partial \psi}{\partial \nabla \rho}\right) \cdot \dot{\bar{\nabla} \rho}+\left(\pi-\frac{\partial \psi}{\partial \rho}\right) \dot{\rho} \geq 0
$$

Then following standard arguments [22], it follows that,

$$
\begin{aligned}
& \frac{\partial \psi}{\partial \dot{\rho}}=0 \Rightarrow \psi=\hat{\psi}(\rho, \nabla \rho) \\
& \chi=\frac{\partial \hat{\psi}(\rho, \nabla \rho)}{\partial \nabla \rho} \\
& \pi(\rho, \nabla \rho, \dot{\rho})-\frac{\partial \hat{\psi}(\rho, \nabla \rho)}{\partial \rho}=\beta \dot{\rho}
\end{aligned}
$$

where to ensure that the dissipation inequality is not violated, the kinetic modulus $\beta$ is given by,

$$
\beta=\hat{\beta}(\rho, \nabla \rho, \dot{\rho}) \geq 0 .
$$

Substituting the micro-force balance into equation (0.6), the evolution of the dislocation density is given by,

$$
\beta \dot{\rho}=\operatorname{div}\left(\frac{\partial \hat{\psi}(\rho, \nabla \rho)}{\partial \nabla \rho}\right)-\frac{\partial \hat{\psi}(\rho, \nabla \rho)}{\partial \rho} .
$$

By an appropriate choice for the free energy, this reduces to the evolution equation developed by Bammann and Aifantis [20] using a form of mixture theory as described previously. As the resolved shear stress on a slip system reaches a critical value, a dislocation can relieve stress by overcoming internal energy barriers and moving along the slip plane. It will continue until it becomes trapped in a new local energy minimum. To describe this process we will follow [23] and assume that the free energy is of the form: 


$$
\psi=\Sigma(\rho)+\frac{1}{2} \Lambda|\nabla \rho|^{2}
$$

The first term will be referred to as the substructure energy and accounts for the creation and annihilation of the dislocation density. The second term, the gradient energy, penalizes non-uniform distributions of dislocations. The importance of the internal stress field arising from gradients in the elastic strain fields of dislocations piled up at boundaries is discussed by Kuhlmann-Wilsdorf. ${ }^{25}$ The evolution of the dislocation density now takes the form,

$$
\beta \dot{\rho}=\frac{\partial \Sigma(\rho)}{\partial \rho}+\operatorname{div}(\Lambda \nabla \rho)
$$

Kocks and Mecking ${ }^{26}$ proposed an evolution equation for the dislocation density based upon the following arguments. As the material deforms, dislocations are stored inversely to their mean free path, which in turn is inversely proportional to the square root of the dislocation density. As deformation continues and the dislocation population grows, the internal stress increases. So does the probability that dislocations will either encounter dislocations of opposite sign and be annihilated or cross slip to a parallel plane and continue slipping. Therefore, the dislocation density evolution is governed by the following equation,

$$
\dot{\rho}=\mathrm{a}_{1} \sqrt{\rho}\left|\dot{\varepsilon}^{\mathrm{p}}\right|-\mathrm{a}_{2} \mathrm{\rho}\left|\dot{\varepsilon}^{\mathrm{p}}\right|
$$

Choosing an appropriate form for the substructure energy $\Sigma(p)$, for the coefficient $\Lambda$, in the gradient energy and for the kinetic modulus $\beta$, equation (0.9) reduces to a modified Kocks-Mecking equation,

$$
\dot{\rho}=c_{1} \nabla^{2} \rho+c_{2} \sqrt{\rho}\left|\dot{\varepsilon}^{\mathrm{p}}\right|-c_{3} \rho\left|\dot{\varepsilon}^{\mathrm{p}}\right|
$$

The coefficient $c_{1}$ represents the mathematical length scale introduced into the continuum, and is related to micro-structural mechanisms. It is interesting to examine other possible functional forms for the free energy. If we assume that the free energy is of the form,

$$
\psi=\Sigma(\rho)+\Lambda \sqrt{\rho} \nabla \rho
$$

then, equation (0.11) takes the form, 


$$
\dot{\rho}=c_{1} \sqrt{\rho} \nabla^{2} \rho+\frac{c_{1}}{2 \sqrt{\rho}} \nabla \rho \cdot \nabla \rho+c_{2} \sqrt{\rho}\left|\dot{\varepsilon}^{\mathrm{p}}\right|-c_{3} \rho\left|\dot{\varepsilon}^{\mathrm{p}}\right|
$$

The length scale in the evolution of the dislocation density now evolves with deformation. The actual physical length scale is probably related to mean free path of the dislocations. In situations where the dislocation substructure determines the mean free path, then the mean free is inversely proportional to the square root of the dislocation density. This is reflected in equation (0.13).

\section{Numerical Implementation}

Constitutive equations having gradient terms in the evolution of state typically have a different mathematical character than evolution equations with only local dependencies (the equations are parabolic partial differential equations instead of hyperbolic ordinary differential equations). Consequently, the numerical solution of the evolution equations with gradient terms involves a somewhat different approach than can be taken with a strictly local theory. For non-local theories, Galerkin finite element formulations are effective for. handling both the spatial and temporal dimensions using the same methodologies employed in solving transient heat conduction problems. The complete solution for the motion and evolution of state involves integrating the evolution equations together with determining the velocity (or displacement) field from the balance laws.

The computation of the velocity field follows precisely the same approach used with a strictly local form of the constitutive theory. A weighted residual on the equilibrium equations is constrained by conservation of mass via a consistent penalty formulation. Because the gradient terms in the evolution of state are restricted to the evolution equations and do not appear in the yield condition, the solution procedure for the velocity field is unaffected by the non-local effects. As constructed, the velocity field at an instant depends on the state, but not on the gradients in state or strain. The motion of the body and the evolution of state are coupled, but for the purposes of simulation are separable. The technique for determining the velocity field may be found in existing references ${ }^{27}$.

\subsection{Hardening}

The modification of the material strength with deformation is embodied in the constitutive model through the evolution of the strength,

$$
\frac{D \kappa}{D t}=\varepsilon_{\kappa} D^{\prime} \nabla^{2} \kappa+H_{\kappa}\left(\kappa, \Phi, D^{\prime}\right)
$$

where $\kappa$ is a measure of the strength of the material and the hardening function, $\mathrm{H}_{\mathrm{K}}$, is written to capture the hardening and recovery processes typical of slip- 
dominated plastic flow. We have developed the non-local capability to work with a number of models having this general form, and have employed a number of those models in this work (see the appendix). The particular model used for each application will be designated with the application, along with appropriate model parameters for the material of interest. For some cases, $\kappa$ has the meaning of strength (controlling the size of the yield surface), while in others we use the dislocation density. In the latter case the strength is assumed to be proportional to the square root of the dislocation density. The numerical procedures for solving equation (0.14) are quite similar whether the variable has the interpretation of strength or dislocation density.

The numerical integration of equation (0.14) is based on a weighted residual formed over the domain as

$$
R_{\kappa}=\int_{\Omega} \Psi_{\kappa}\left[\frac{D \kappa}{D t}-\varepsilon_{\kappa} D^{\prime} \nabla^{2} \kappa-H_{\kappa}\left(\kappa, \Phi, D^{\prime}\right)\right] d \Omega
$$

where $\Psi_{\mathrm{K}}$ is a set of scalar weighting functions and $\Omega$ is the volume of the body. Following standard finite element practices [8], the continuity requirements on the trial functions used to represent the dislocation density are shifted to the weighting functions through integration by parts, assuming sufficient interelement continuity. This gives, after application of the divergence theorem

$$
\mathrm{R}_{\kappa}=\int_{\Omega} \Psi_{\kappa} \frac{\mathrm{DK}}{\mathrm{Dt}} \mathrm{d} \Omega+\int_{\Omega} \varepsilon_{\kappa} \mathrm{D}^{\prime} \nabla^{2} \kappa \nabla \Psi_{\kappa} \mathrm{d} \Omega-\int_{\Omega} \Psi_{\kappa} H_{\kappa}\left(\kappa, \Phi, D^{\prime}\right) \mathrm{d} \Omega-\int_{\Gamma^{1}} \Psi_{\kappa} \mathrm{f}_{\kappa} \mathrm{d} \Gamma
$$

where $\Gamma$ is the boundary of $\Omega$, and $\Gamma^{1}$ is that portion of the boundary where the dislocation density gradient is known.

$$
\varepsilon_{\kappa} D^{\prime} \nabla \kappa \cdot n=f_{\kappa}
$$

The remainder of the surface is denoted $\Gamma^{2}$ with the boundary condition that

$$
\kappa=\bar{\kappa}
$$

This latter condition is not encountered in the applications discussed later, however.

We next introduce into the weak form of the evolution equation the temporal discretization for the strength as

$$
\kappa=\frac{\left(\kappa_{t}+\kappa_{t-\delta t}\right)}{2} \bar{\kappa} \quad \dot{\kappa}=\frac{\left(\kappa_{t}-\kappa_{t-\delta t}\right)}{\Delta t}
$$


and finite element interpolation for the trial and weight functions as

$$
\kappa=[N]\{K\} \quad \Psi_{\kappa}=[N]\{S\}
$$

where $[N]$ are the interpolation functions, $\{K\}$ are nodal values of the strength, and $\{S\}$ are nodal values of the weights. We assemble elemental contributions to $R_{K}$ and require that it vanish for arbitrary values of the weights equation, giving a matrix equation for the nodal values of the strength at the end of each time interval based on the values at the beginning of the interval and the rate of strain hardening during the interval.

$$
\left[\mathrm{C}_{1}\right]\{K\}_{\mathrm{t}}=\left[\mathrm{C}_{2}\right]\{K\}_{\mathrm{t}-\delta \mathrm{t}}+\left\{\mathrm{F}_{\mathrm{K}}\right\}
$$

where for a single element

$$
\begin{aligned}
& {\left[\mathrm{C}_{1}\right]=2 \int_{\Omega_{e}}[\mathrm{~B}]^{\top} \varepsilon_{\kappa}[\mathrm{B}] \mathrm{d} \Omega+\delta \mathrm{t} \int_{\Omega_{e}}[\mathrm{~N}]^{\top}[\mathrm{N}] \mathrm{d} \Omega} \\
& {\left[\mathrm{C}_{2}\right]=-2 \int_{\Omega_{e}}[\mathrm{~B}]^{\top} \varepsilon_{\kappa}[\mathrm{B}] \mathrm{d} \Omega+\delta \mathrm{t} \iint_{\Omega_{e}}[\mathrm{~N}]^{\top}[\mathrm{N}] \mathrm{d} \Omega} \\
& \left\{\mathrm{F}_{\mathrm{K}}\right\}=2 \delta \mathrm{t} \int_{\Omega_{e}}[\mathrm{~N}]^{\mathrm{T}} \mathrm{H}_{\kappa}\left(\kappa, \Phi, D^{\prime}\right) \mathrm{d} \Omega+\delta \mathrm{t} \int_{\Gamma_{e}}[\mathrm{~N}]^{\mathrm{T}} \mathrm{f}_{\kappa} \mathrm{d} \Gamma
\end{aligned}
$$

and $[\mathrm{B}]$ contains the spatial derivatives of $[\mathrm{N}]$.

\subsection{Damage}

The rate of growth of damage may depend on the gradients of damage in the same manner that the strength evolution depends on it gradients. Introducing a gradient dependence, the ductile void growth is described by an equation of the form

$$
\frac{\mathrm{d} \Phi}{\mathrm{dt}}=\varepsilon_{\Phi} \mathrm{D}^{\prime} \nabla^{2} \Phi+\mathrm{H}_{\Phi}\left(\kappa, \Phi, \mathrm{D}^{\prime}\right)
$$

where on a portion of the surface, designated $\Gamma^{1}$,we specify

$$
\varepsilon_{\Phi} D^{\prime} \nabla \Phi \cdot \mathrm{n}=\mathrm{f}_{\Phi}
$$

The remainder of the surface is denoted $\Gamma^{2}$ with the boundary condition that

$$
\Phi=\bar{\Phi}
$$


The function $\mathrm{H}_{\Phi}$ is specified by the material model chosen for the analysis, and is given in the appendix for the applications studied here. A residual is formed from equation (0.23) as

$$
\mathrm{R}_{\Phi}=\int_{\Omega} \Psi_{\Phi}\left[\frac{\mathrm{D} \Phi}{\mathrm{Dt}}-\mathrm{H}_{\Phi}\left(\kappa, \Phi, \mathrm{D}^{\prime}\right)\right] \mathrm{d} \Omega
$$

where $\Psi_{\Phi}$ is a set of scalar weighting functions. Again, we introduce into the weak form of the evolution equation the temporal discretization for the void volume as

$$
\Phi=\frac{\left(\Phi_{\mathrm{t}}+\Phi_{\mathrm{t}-\delta \mathrm{t}}\right)}{2} \dot{\Phi}=\frac{\left(\Phi_{\mathrm{t}}-\Phi_{\mathrm{t}-\delta \mathrm{t}}\right)}{\delta \mathrm{t}}
$$

and finite element interpolation for the trial and weight functions as

$$
\Phi=[\mathrm{N}]\{\oplus\} \quad \Psi_{\Phi}=[\mathrm{N}]\{S\}
$$

where $\{\oplus\}$ are nodal values of the strength, and $\{S\}$ are nodal values of the weights. As with the formulation for the evolution of the strength, we require the residual vanish for arbitrary values of the weights equation, giving a matrix equation for the nodal values of the void volume at the end of each step in terms of values at the start of the step.

$$
\left[\mathrm{C}_{3}\right]\{\oplus\}_{\mathrm{t}}=\left[\mathrm{C}_{4}\right]\{\oplus\}_{\mathrm{t}-\delta \mathrm{t}}+\left\{\mathrm{F}_{\Phi}\right\}
$$

where for a single element

$$
\begin{aligned}
& {\left[\mathrm{C}_{3}\right]=2 \int_{\Omega_{e}}[\mathrm{~B}]^{\top} \varepsilon_{\Phi}[\mathrm{B}] \mathrm{d} \Omega+\int_{\Omega_{e}}[\mathrm{~N}]^{\top}[\mathrm{N}] \mathrm{d} \Omega} \\
& {\left[\mathrm{C}_{4}\right]=-2 \int_{\Omega_{e}}[\mathrm{~B}]^{\top} \varepsilon_{\Phi}[\mathrm{B}] \mathrm{d} \Omega+\int_{\Omega_{e}}[\mathrm{~N}]^{\top}[\mathrm{N}] \mathrm{d} \Omega} \\
& \left\{\mathrm{F}_{\Phi}\right\}=2 \delta \mathrm{t} \int_{\Omega_{e}}[\mathrm{~N}]^{\top} \mathrm{H}_{\Phi}\left(\kappa, \Phi, \mathrm{D}^{\prime}\right) \mathrm{d} \Omega+\int_{\Gamma_{e}}[N]^{\top} \mathrm{f}_{\mathrm{p}} h i d \Gamma
\end{aligned}
$$

and $[B]$ again contains the spatial derivatives of $[N]$. 


\section{Applications}

\subsection{Hardening Gradients and Stability}

\subsubsection{Overview}

In finite element simulations of mechanical boundary value problems, stability is characterized by a loss of homogeneity of the deformation field. Non-local theories don't play a role as long as state is uniform, since there are no gradients to influence the hardening. Once a perturbation exists, however, non-local theory is important because it will influence the hardening rates. In each of the following examples, the non-uniformity of deformation fields results in situations where the gradient terms have a strong influence on the final state. In addition, the introduction of the gradient terms results in valid in the post bifurcation regime, at which point the boundary value problem would become ill posed without the introduction of the gradient terms.

\subsubsection{Variable Threshold Rod Analyses}

\section{Description}

The variable threshold rod experiment was designed so that a spatially varying deformation field would occur in a specimen with simple boundary conditions Figure 1. The intent is to able to study the fidelity of constitutive models for the bulk material without uncertainties being introduced by imprecise knowledge of the boundary conditions. In this case, the experiment is simply a tension test, but with a tensile specimen that has strength that depends on the position along its gage length. Under uniaxial tension, the specimen deforms differently along its length owing to this spatial variation in its strength. Data for the deformation of specimens with both straight and tapered crossections has been published, and previously was used to explore issues of how effectively a back stress could account for changes in the loading direction. Here, we examined the impact that the non-local hardening theory has on the evolution of deformation, especially the propensity to neck. It should be noted that both the straight and tapered rods do neck with continued extension, but the distribution of strains along the gage length are sensitive to details of the hardening model, and thus to the inclusion of a non-local effect in the hardening. 


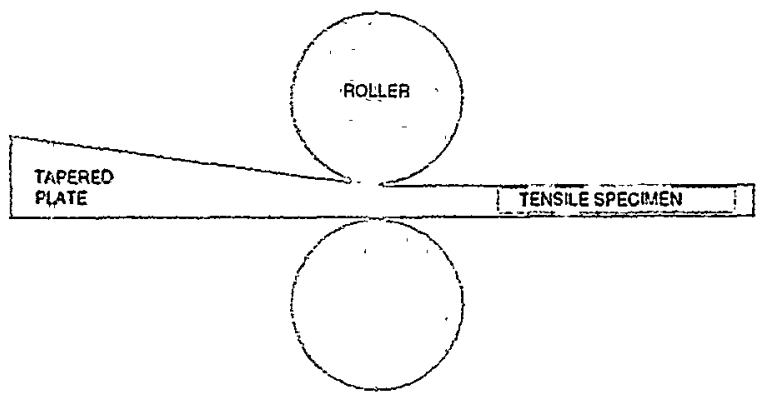

Figure 1. Schematic of the variable threshold rod. A tapered plate is rolled flat and a tensile specimen with non-uniform properties is extracted.

\section{Simulations}

The specimen was discretized with the same finite element mesh as was used in the prior study ${ }^{28}$ and loaded in the same manner with an imposed axial velocity on one end and fixed position at the other end.

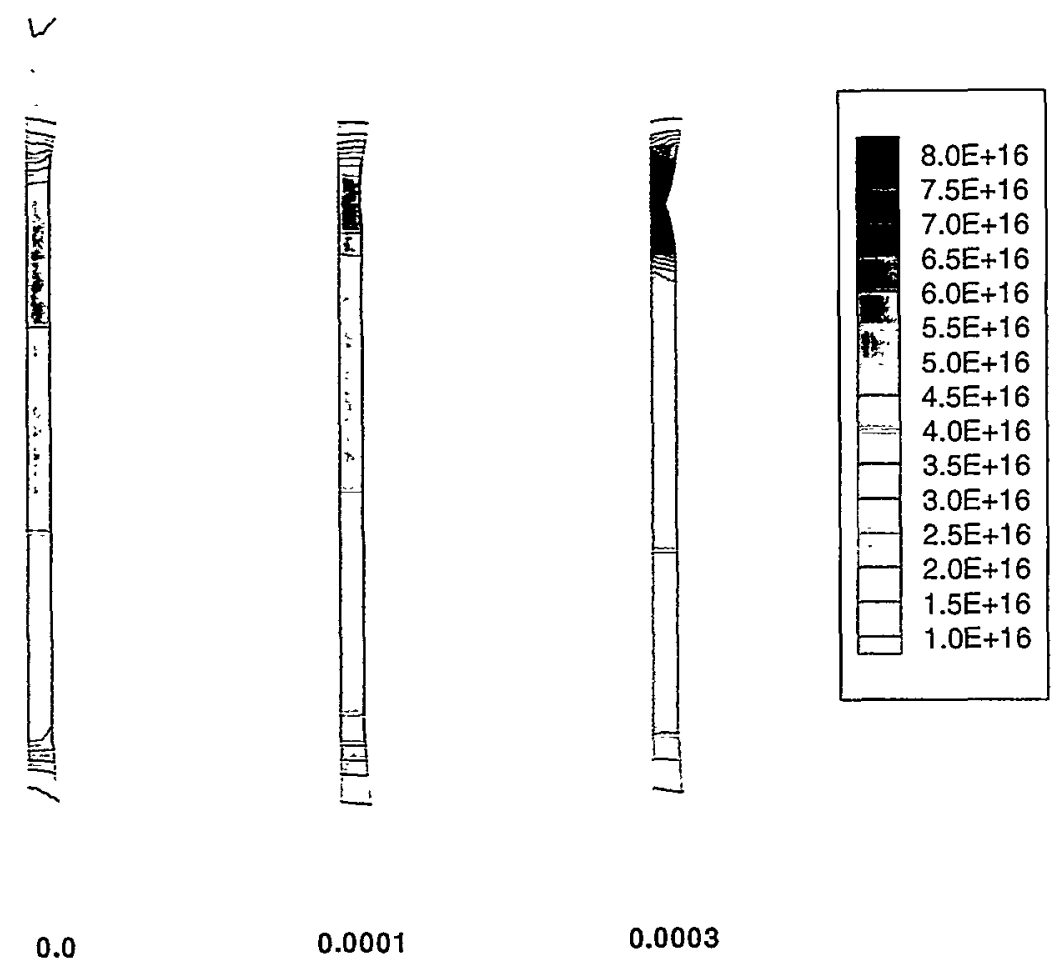

Figure 2. Deformed geometries for various non-local parameters. Values of $\varepsilon_{\mathrm{r}}$ have units of $\mathrm{m}^{2}$.

At extensions corresponding to those at which diameter measurements were taken in the experiments, diameter values in the simulations were saved, along with the distributions of dislocation density. 
The material in the experiments was copper, and for the simulations parameters for the constitutive model were obtained by fitting to compression data. In contrast to the prior study, here only a single scalar hardening variable was, which is accomplished by requiring that the tensor back stress remain fixed at zero. The scalar variable is the dislocation density; the equation for its evolution and the parameters for copper are given in the appendix.

The initial distribution of the scalar value was estimated by integrating its evolution equation over a plane strain compression path corresponding to the rolling reduction used to induce the starting variation in state, in the copper material. This distribution was imposed on the rod to initialize the state for the simulations of the tensile loading. The initial values of the scalar state variable are given in Table 3.

\section{Results}

Three simulations are presented from those performed. These have values of the non-local parameter ranging from 0 to $3 \times 10^{-4} \mathrm{~m}^{2}$. The value of 0 corresponds to the local theory; the value of $3 \times 10^{-4} \mathrm{~m}^{2}$ invokes a strong effect of the non-local contribution in the evolution. The deformed geometries for the three cases are shown in Figure 2 at the point where the total extension in all of the cases correspond to the last interruption in the experiment. There is a strong tendency for the rod to neck at less extension with higher values of the non-local parameter. This is due to the suppression of hardening in the region where the neck forms as a consequence of the diffusion of the dislocation density. In the case corresponding to the local theory, the material hardening is greater in the regions where the deformation is greater. This tends to stabilize the deformation by shifting the deformation to adjoining regions. In contrast, with the non-local contribution, the regions of greatest deformation continue to experience deformation since the diffusion of dislocations from the regions of high dislocation density mitigates the stabilizing effect of higher strength. This is evident in the plots from the more diffuse dislocation density fields with larger non-local parameter.

\subsubsection{Plate with Array of Holes}

\section{Description}

As part of the project, a sequence of tests was performed on copper specimens made from sheets with arrays of holes. Several sheet thicknesses were considered, and for each, the hole array geometry was specified so that geometric similarity was retained over all of the specimens. The tests are described in Section 6. The simulations presented here were performed as precursors to these experiments to provide guidance for the experimental design.

The geometry in the simulations was modeled after that used in a study by Tvergaard and Needleman [16]. A plate containing an array of holes as shown 
in Figure 3 was loaded such that one in-plane dimension of the plate extended and the other compressed. The deformation between the holes is quite nonuniform, even though the boundary conditions would correspond to a homogeneous deformation in a solid plate (one without the array of holes). The non-uniformity of deformation gives rise to a localization of straining into bands that connect the holes. Our interest was in understanding the sensitivity of band formation to the strength of the non-local contribution in the equation for the evolution of the dislocation density.

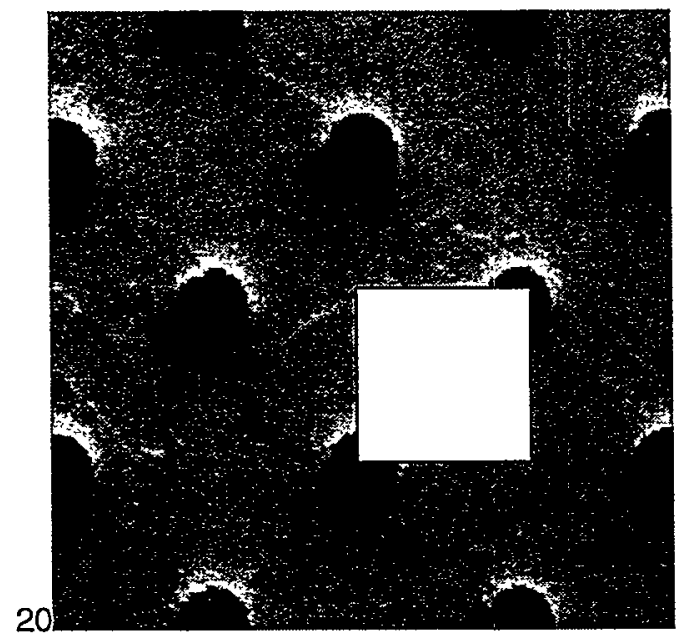

Figure 3. Schematic of plate with array of holes showing unit cell.

\section{Simulations}

A unit cell of the array of hole was discretized with finite elements. The unit cell is defined by two mirror planes in the array, one constituting a reflection about the $x$-axis and one about the $y$-axis. Further reductions in the cell are not possible because the cell does not have equal dimensions in the $x$ and $y$ directions. Loading consisted on applying a uniform velocity to one of the surfaces with a $\mathrm{x}$-direction normal, and a uniform velocity to one of the surfaces having a $y$-direction normal. The other surfaces where constrained to have zero velocity in the direction normal to the surface, except for the hole surfaces, which were traction free.

Simulations were performed using parameter sets for aluminum and copper. Here, we discuss only the copper results, as those correspond to the material chosen for the experiments. The hardening is based on the evolution of the dislocation density using the evolution equation given in the appendix. The parameter set for the copper is based on compression tests, and is given in Table 3. As with the variable property rod discussed in Section 5.1.2 three values of the non-local hardening parameter were specified. Values were 
chosen to produce conditions ranging from no non-local effects (local theory) to relatively strong non-local influence, in which $3 \times 10^{-4} \mathrm{~m}^{2}$.

\section{Results}

The simulations exhibit the tendency to form bands of straining that connect the holes. The strength of the bands is dependent on the level of the non-local contribution to the hardening evolution equation. In general, the greater the nonlocal parameter, the greater the tendency for the strains to localize. This is shown in Figure 4 the case of low non-local parameter value and in Figure 5 for high non-local parameter. As with the variable property rod, the localized

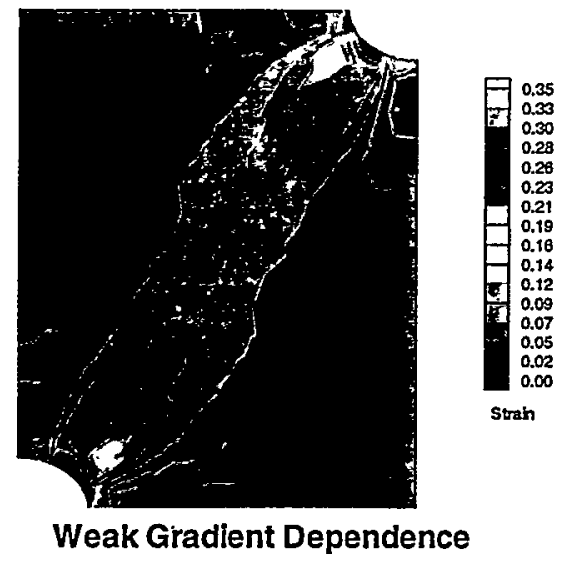

Figure 4. Distribution of effective strain.

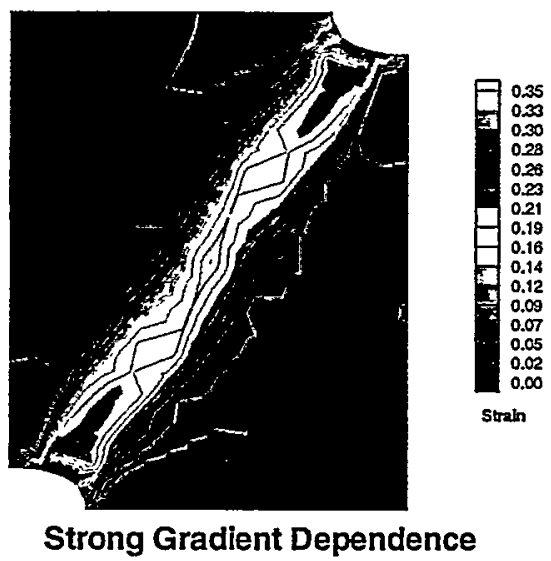

Figure 5. Distribution of effective strain.

straining is mitigated without the non-local contribution because the straining in the zone of high deformation elevates the strength locally and inhibits further straining there. The impact of the non-local contribution is to spread the dislocation density to neighboring regions, so there is less strength differential between the regions that deform to greater or lesser extents. The dislocation density distribution is shown in Figure 6 and Figure 7 for the case of low and high non-local parameter values, respectively. This acts to continue the deformations in the same volume and leads to more highly developed bands. 


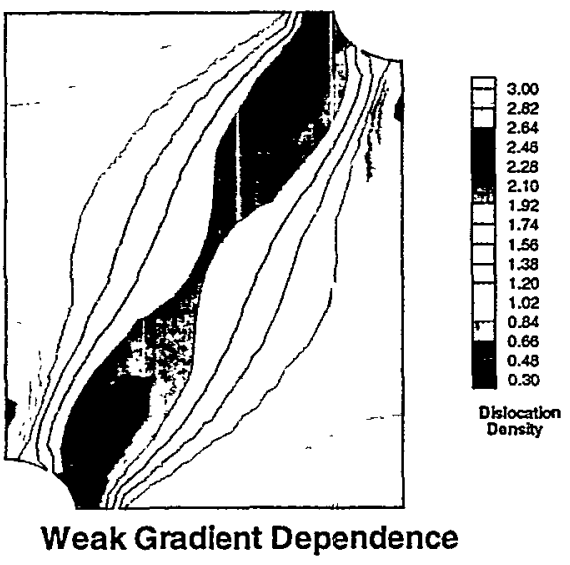

Figure 6. Distribution of dislocation density for small gradient term.

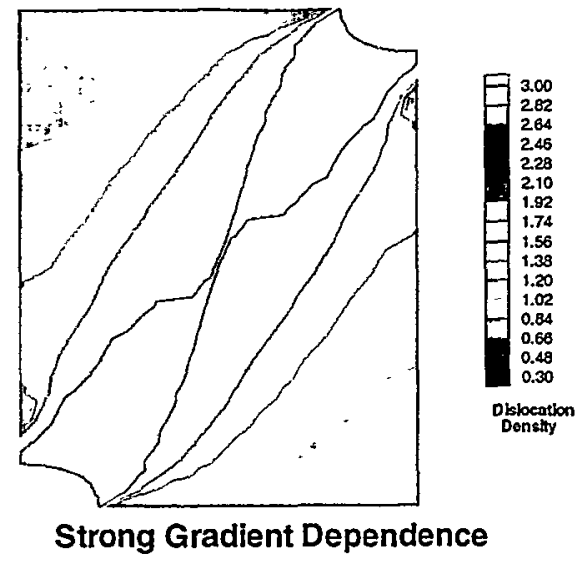

Figure 7. Distribution of dislocation density for large gradient term.

\subsubsection{Discussion}

It has been illustrated that the gradient term plays a strong role in localization of straining. The effect of the gradient term is to slow apparent hardening where the deformation is high, and to speed the apparent hardening where deformation is low, in comparison to a local model. The effect is important is computing localization both because the theory introduces a length scale (and thus some degree of mesh independence) and because it portrays a different physical system than local systems can depict.

\subsection{Hardening Gradients and Damage}

\subsubsection{Overview}

In this study we have examined the influence of gradient terms in both the damage evolution and the hardening equations. Gradient dependence in the evolution of damage can act to localize damage more rapidly or to diffuse peak values depending on the sign used for the non-local parameter. A negative nonlocal parameter causes localization, mimicking coalescence. That is, volumes of high damage are driven towards each other. Ramaswamy Aravas [15] have utilized this type of model to investigate mesh dependency of solutions. A positive non-local parameter can also assist numerically by decreasing the mesh dependency. The non-local terms in the hardening also influence the damage evolution rate through the dependence that the void growth rate has on the strength of the material. 


\subsubsection{Damage Simulations}

Void growth during the extension of a notched rod was studied previously ${ }^{29}$ using the theory presented here, with the exception of the gradient term appearing in the evolution equation for the strength (Equation (0.23). We return to that application to demonstrate the strong influence that gradients in the strength have on the void growth. The axi-symmetric specimen shown in Figure 8 is pulled in tension with a fixed crosshead speed of

$2.1167 \times 10^{-5} \mathrm{~m}^{2}$ for a total of $200 \mathrm{~s}$.

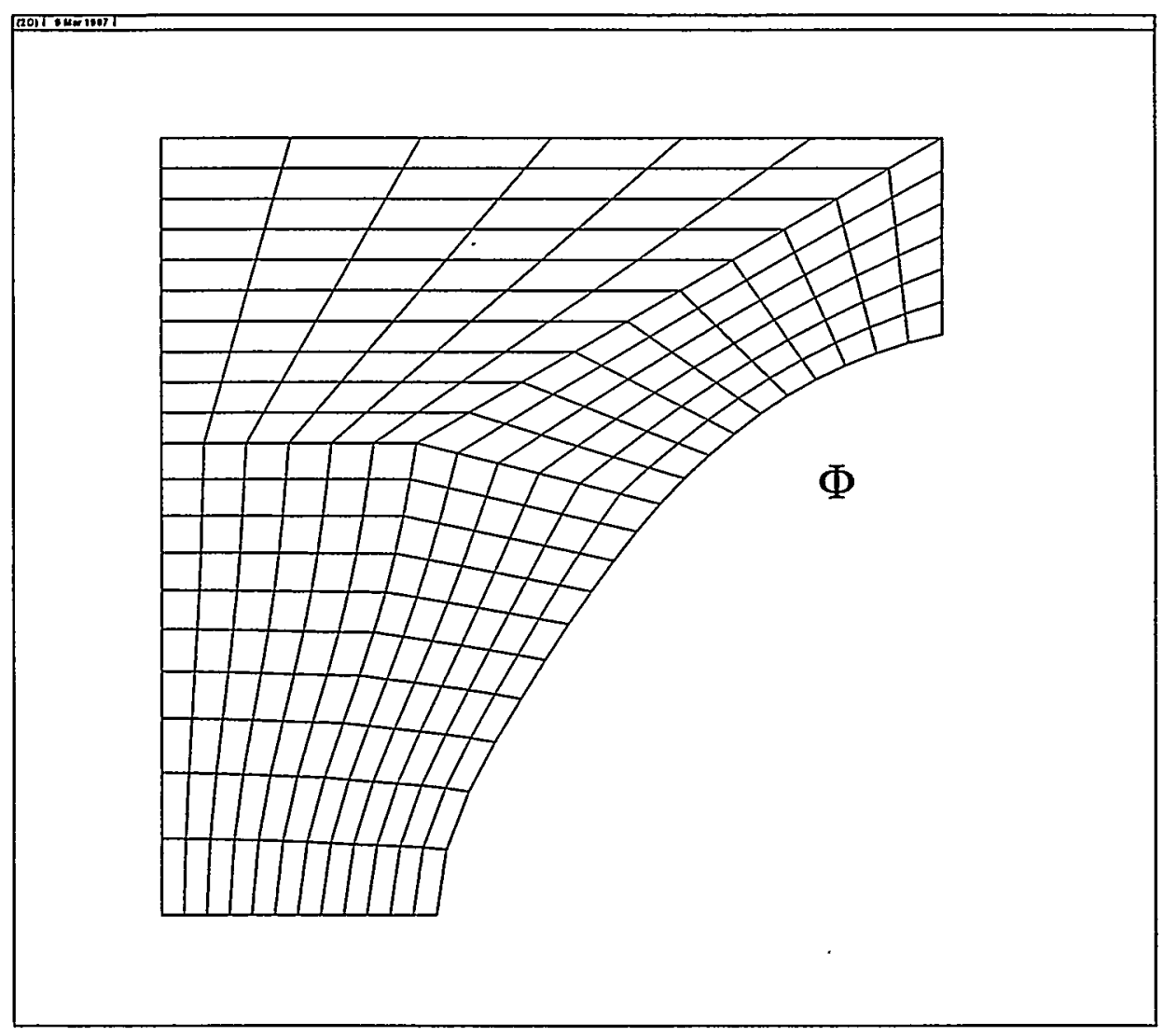

Figure 8. Finite element mesh for notched specimen simulations.

The initial strength is $64 \mathrm{MPa}$ and the initial void fraction is 0.0011 . The temperature initially is $373 \mathrm{~K}$, and because the test is conducted very slowly, isothermal conditions are assumed to persist throughout the test. Parameters for both the plastic flow model and the void growth model are given in Tables 3 and 4.

To illustrate the impact of the gradient term in the hardening, simulations performed with two different values of $\varepsilon_{\mathrm{K}}$ are compared to the original formulation in which no dependence on the gradients appears. Shown in Figure 9 and Figure 10 are the strength and void fractions corresponding to the case of 
$\varepsilon_{\mathrm{K}}$ of zero at the end of the loading. The strength is elevated by the largest amount in a band across the smallest cross section where the straining is most intense. The peak value of koccurs within this band and is approximately 128 $\mathrm{MPa}$. The void fraction exhibits the greatest rise along the centerline and the minimum cross section, as reported previously [29]. The void fraction reaches a maximum value of about 0.01 in this case.

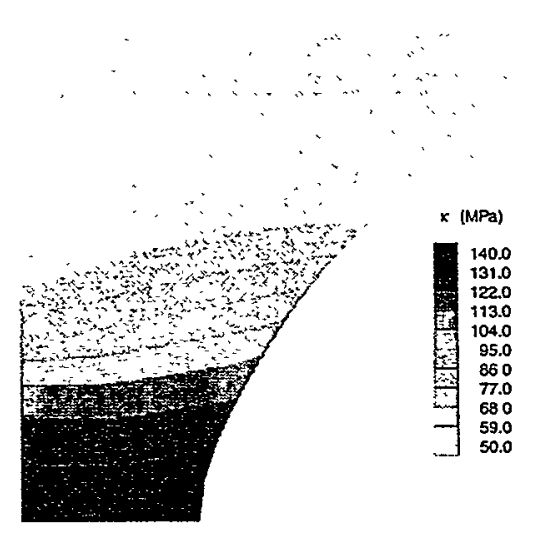

Figure 9. Distribution of strength in the notched zone at the end of loading for $\varepsilon_{\mathrm{K}}$ of 0.0 . Peak value - 128 MPa.
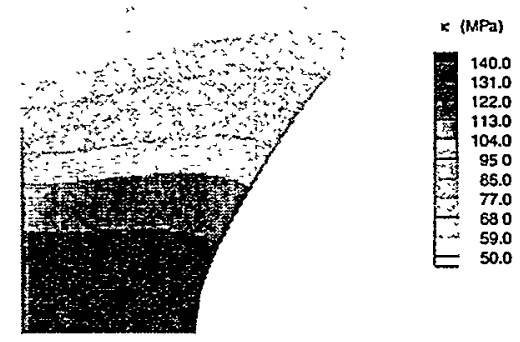

Figure 11. Distribution of strength in the notched zone at the end of loading for $\varepsilon_{\mathrm{K}}$ of 0.0001 . Peak value $-118 \mathrm{MPa}$.

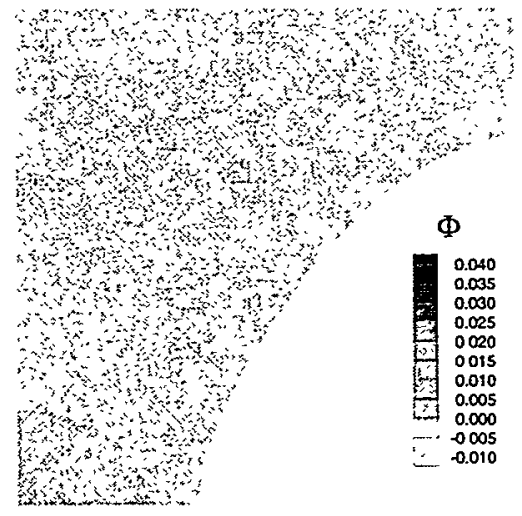

Figure 10. Distribution of void fraction in the notched zone at the end of loading for $\varepsilon_{\mathrm{K}}$ of 0.0 . Peak value -0.010

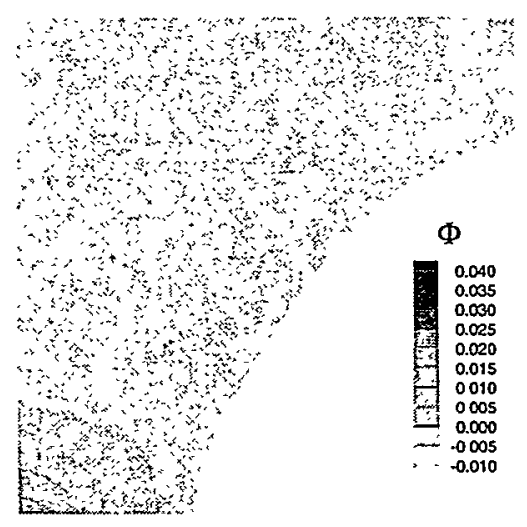

Figure 12. Distribution of void fraction in the notched zone at the end of loading for $\varepsilon_{\mathrm{K}}$ of 0.0001 . Peak value -0.024 . 


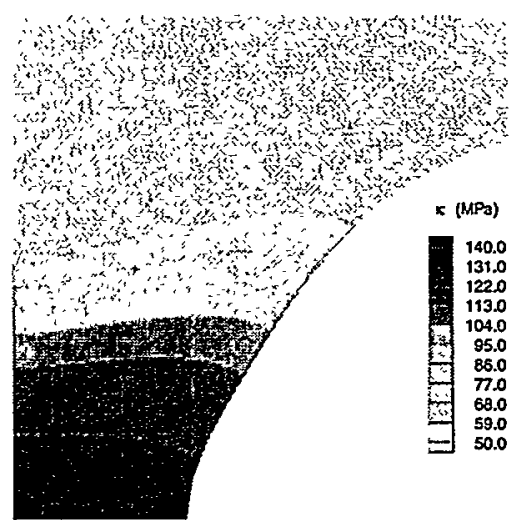

Figure 13. Distribution of strength in the notched zone at the end of loading for $\varepsilon_{\mathrm{K}}$ of 0.0003 . Peak value $-115 \mathrm{MPa}$.

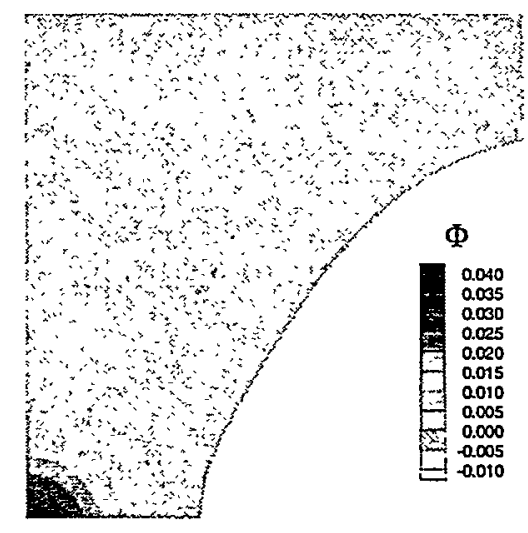

Figure 14. Distribution of void fraction in the notched zone at the end of loading for $\varepsilon_{\mathrm{k}}$ of 0.0003 . Peak value $\mathbf{0} \mathbf{0 . 0 3 6}$.

The effects of the gradient term in the evolution of kare shown for two values of $\varepsilon_{\mathrm{K}}: 0.0001$ and 0.0003 . Shown in Figure 11 and Figure 12 are the strength and void fraction distributions corresponding to the end of the loading for the case of $\varepsilon_{\mathrm{K}}$ of 0.0001 . Note that the while the strength distribution is qualitatively similar to the case in which $\varepsilon_{\mathrm{K}}$ is zero, the peak value is lower $(118 \mathrm{MPa})$ and the gradients near the minimum cross section are weaker. The corresponding void fraction distribution shows maximum values more than double $\left(\Phi_{\max }=0.024\right)$ those predicted in the zero $\varepsilon_{\mathrm{K}}$ case.

The differences in the responses between $\varepsilon_{\mathrm{K}}$ of 0.0 and 0.0001 are accentuated for $\varepsilon_{\mathrm{K}}$ of 0.0003 . These appear in Figure 13 and Figure 14 for the strength and void fraction, respectively. The strength gradients are suppressed near the minimum cross section and the peak value is the lowest of the three cases considered. The void fraction, on the other hand, is approximately three times the result obtained using no gradient term in the evolution $\left(\Phi_{\max }=0.036\right)$.

The influence of gradients in the evolution of the strength can be seen to have a pronounced effect on the growth of voids. This occurs here because the peak value of the mean stress occurs at the same spatial location as the highest strength. Such an occurrence is clearly not unusual, as it implies that both the mean stress and the deviatoric stress are high at the same spatial location. It has a strong effect because the void growth rate is quite sensitive to the ratio of the mean stress to the strength. The effect of the gradient term is to diffuse the 
peak values of strength, which results in higher values of this ratio and therefore higher void growth rates.

\subsubsection{Discussion}

With the current model, void growth rates depend strongly on strength. Spatially, high mean stress and high deformations (deviatoric stresses) occur in the same region. The non-local terms influence the mean stress to strength ratio, and thus the damage evolution rate. Therefore, small differences in strength have strong influence on damage growth due to exponential dependence on ratio.

\subsection{Hardening Gradients and Hardness Profiles}

\subsubsection{Overview}

In the following we are interested in the issue of enhanced hardening due to small scale. At a very small length scale, local continuum theory can not accurately predict observed experimental results. In the following we examine two sets of published data in which local continuum theory fails to capture observed phenomena, and attempt to capture the observed response using gradient terms in the hardening evolution. In particular, we examine the torsion of very thin wires [3], where it has been observed that at very small wire diameters, the flow stress increases with decreasing diameter which is inconsistent with local continuum theory. Similarly, it has been reported that during friction ${ }^{30}$, the dislocation structure propagates more deeply into the material that predicted by local theory and the flow stress near the surface is much higher that predictions of local continuum theory. We investigate the importance of gradient terms in the hardening evolution in resolving these apparent anomalies.

\subsubsection{Torsion of Fine Wires}

\section{Description}

Experiments conducted on the torsion of fine wires [3] reported an apparent elevation in the strength of wires with smaller diameter. The data motivated the specification of a yield strength as a function of the gradient in strain in addition to other commonly used correlations. Another approach to modeling the observed behavior would be to alter the evolution equations for the state in a manner that gives a dependence on the magnitude of gradients in the field variables. This is, of course, the methodology being investigated in this project. The experiment thus provides another test case to investigate. In particular, we examined the elevation in strength in wires of various dimensions due deformation caused by an applied twist as shown in figures 15 and 16 . Hardening formulations with and without non-local dependencies were examined and the resulting estimates for the wire strength compared to the corresponding experimental records. 


\section{Simulations}

Analyses were performed in which the deformation is imposed and the evolution of state is simulated by integrating the appropriate hardening equations. In simple torsion, the circumferential velocity component varies linearly with radial and axial location, giving one non-zero deformation rate component $D_{z \theta}=c r$ where $c$ is a constant) and an effective deformation rate that is proportional to $D_{z \theta}$. With the kinematics imposed, the evolution equations are integrated directly since the deformation rate that enters the evolution equations may be written as a function of the position and the state:

$$
\frac{\partial \rho}{\partial \mathrm{t}}=\varepsilon_{\rho}\left(\frac{1}{\mathrm{r}} \frac{\partial}{\partial \mathrm{r}}\left(\mathrm{r} \frac{\partial \rho}{\partial \mathrm{r}}\right)\right)+\left(\frac{\mathrm{H}}{\sqrt{\rho}}-\mathrm{R}_{\mathrm{D}}\right) \rho \sqrt{\frac{2}{3}} \mathrm{Cr}
$$

with boundary conditions specified as

$$
|\nabla \rho|=0 \text { at } r=0
$$

and

$$
\varepsilon_{\rho}|\nabla \rho|=f(\rho, r) \text { at } \quad r=r_{0}
$$

The hardening coefficient $\mathrm{H}$ was considered to be dependent on the gradient of the dislocation density itself, with higher hardening rates arising from higher values of the gradient according to:

$$
\mathrm{H}=\mathrm{H}_{0}\left(1+\left|\frac{\nabla \rho}{c_{1}}\right|^{c_{2}}\right)
$$

This set of equations was solved using a one-dimensional reduction of the formulation summarized in Section 4.

\section{Results}

The applied kinematics impose gradients in the deformation rate, and consequently drive the formation of gradients in the dislocation density. The elevation of strength by strain hardening can be influenced by the gradients of dislocation in the model here either through the diffusion term, $\varepsilon_{\rho} \nabla \rho$, or through the hardening coefficient, $\mathrm{H}$. The former cause does not act in the correct direction to result in increased strength with smaller wire diameter. Elevating the strength of the outermost fibers of a wire has the greatest impact on increasing the torque required to twist it. Straining is greatest at the outside radius of the wire, and in a local theory the strain hardening in greatest there. The diffusion term tends to diminish the dislocation density at the outside radius and 
homogenize the dislocation density more effectively in smaller diameter wires than in larger ones. Thus, the diffusion term contributes to an apparent softening of the wires with smaller gauge.

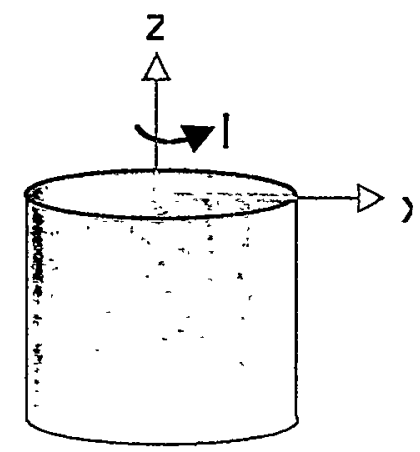

Figure 15. Schematic of wire.

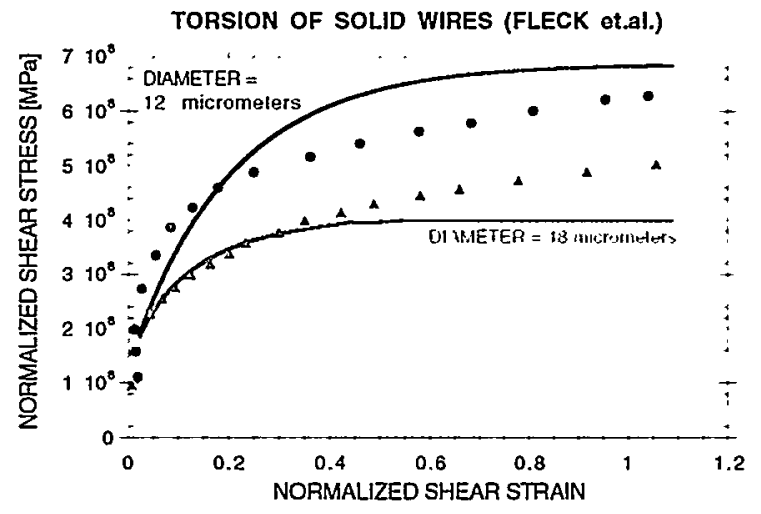

Figure 16. Model versus experiment for various diameter wires.

On the other hand, introduction of the dependence of the hardening coefficient, $\mathrm{H}$, on the gradient of the dislocation density does serve to enhance hardening and its spatial distribution in a way that elevates the strength of wires smaller gauges in comparison to those of larger gauge, as reported for the experiments. Comparison to the data reported by Fleck [3] is shown in Figure 16. Although the magnitude of the gradient increases with radius, giving the outside fibers enhanced level of the dislocation density, the effect is greater for smaller diameters. This produces greater torque for equivalent twist as the wire gauge decreases.

\subsubsection{Friction Analyses}

\section{Description}

Frictional tractions often are associated with considerable near surface plastic straining. Due to the roughness of surfaces, the actual contact area between two bodies usually is much less than the nominal (apparent) area of contact. Even under moderate load levels, stresses at points of contact can be sufficient to induce plastic straining. Published experiments show influence of the near surface deformations extending to depths in the material that are several times the characteristic asperity height. Recent experiments [30] have formulated correlations between measures of strain, dislocation structure, and flow stress. The elevation of flow stress appears to a greater depth than is expected from local theories of strain hardening. In these simulations we examine the influence of a non-local contribution on the depth of hardening, and compare to the experimental correlations. 


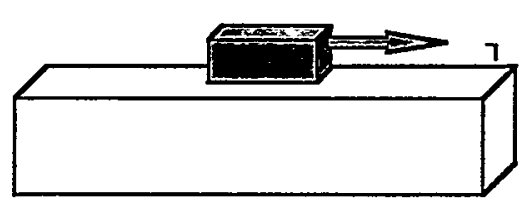

Figure 17. Schematic of friction test.

\section{Simulations}

The experiments consist of sliding a copper block between steel plattens as shown in Figure 17. In the analysis, a single asperity is considered having a ratio of actual contact area to apparent contact area of one-third. The asperity height, as measured by the difference between the highest and lowest points on the surface, is $2 \mu \mathrm{m}$ the asperity width is $33.3 \mu \mathrm{m}$. A planar geometry is assumed (consistent with the lay of the asperities), and a plane strain deformation mode is imposed. The domain discretized with finite elements extends $50 \mu \mathrm{m}$ below the top of the asperity, and $33.3 \mu \mathrm{m}$ to each side of the asperity. The full domain is $100 \mu \mathrm{m}$ wide and $50 \mu \mathrm{m}$ deep. Along the asperity surface, a tangential velocity of $100 \mu \mathrm{m}$ is imposed together with a normal traction. The normal tractions took on values corresponding to the experiments [30], with values ranging between 12 $\mathrm{MPa}$ and $22 \mathrm{MPa}$, based on the nominal contact area.

The material in the experiments is copper, which was modeled in the simulations with the hardening based on elevation of the dislocation density (see the appendix for the evolution equation). Parameter values are given in Table 3. A range in values of the non-local parameter were considered spanning 0 to $5 \times 10^{-6} \mathrm{~m}^{2}$ which gave is sufficient to observe a qualitative change in the dislocation density distributions following the shearing invoked by the asperity loading.

\section{Results}

Figures 18-21 depict the deformation rate for low and high normal stresses for two values of the non-local parameter, $1 \times 10^{-7} \mathrm{~m}^{2}$ and $35 \times 10^{-6} \mathrm{~m}^{2}$ The strong trend to localize the straining in a narrow band immediately below the asperity surface is evident. 
High Normal Pressure

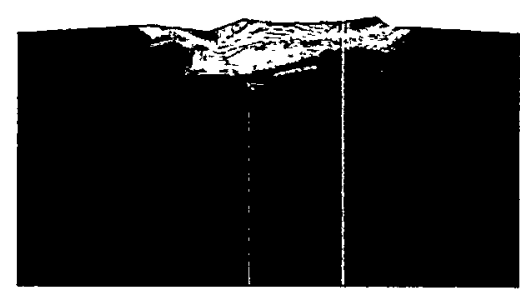

Strong Gradient Dependence

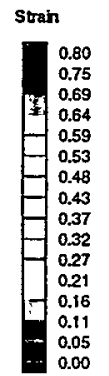

Figure 18. Strain-rate distribution with high stress and strong gradient
High Normal Pressure

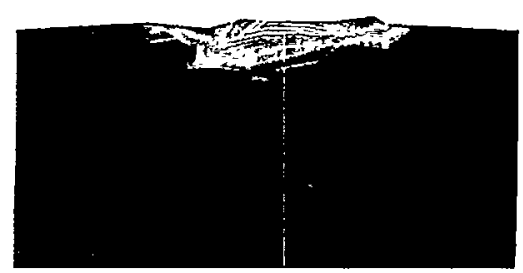

Weak Gradient Dependence

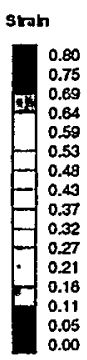

Figure 19. Strain-rate distribution with high stress and weak gradient

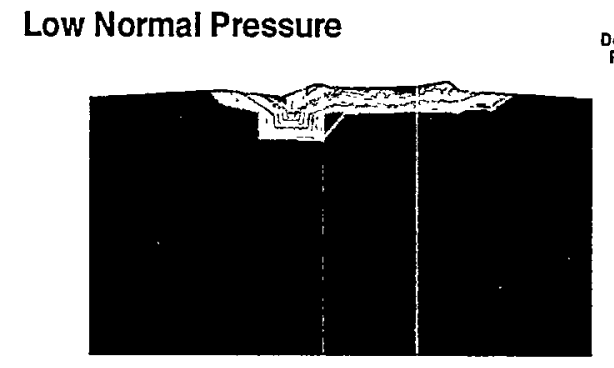

Strong Gradient Dependence
Deformation
Rate $(1 / 3)$

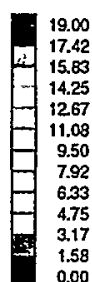

Figure 20. Strain-rate distribution low normal with stress and strong gradient.
Low Normal Pressure

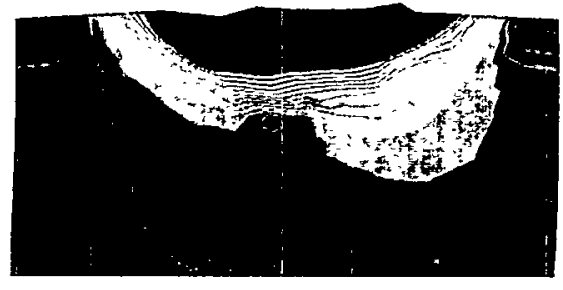

Weak Gradient Dependence

Figure 21. Strain-rate distribution with low normal stress and weak gradient.

The shearing of an asperity strain hardens material in the neighborhood of the asperity. According to a local theory, the hardening remains confined to the material that is deformed, which for this application is a narrow zone directly below the asperity. The inclusion of a non-local contribution has the effect of spreading the hardened region to a zone deeper in the material, and smoothing the distribution of dislocation density in the highly deformed region immediately below the surface. Figures 22-25 show the dislocation density distributions. Two features of the distributions can be noted. First, the overall level of the dislocation density is suppressed by the introduction of the non-local contribution. Peak values of dislocation density are substantially lower, and correspondingly, the magnitude of the tangential traction is lower. Secondly, the introduction of a non-local contribution results in a more uniformly hardened region extending more deeply in the material even while restricting the deformations to remain close to the surface. This in a similar behavior to that seen in the plate with holes and variable property rod applications discussed earlier. By limiting the growth in the dislocation in highly deforming regions, the tendency to shift the deformation to less hardened regions is diminished. Thus, hardening can occur to greater depths without requiring that the deformation occur proportionally. 
High Normal Pressure

Dhelocation

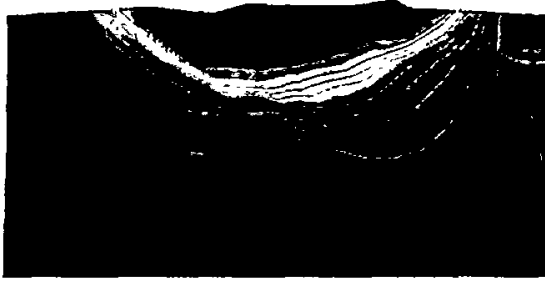

Weak Gradient Dependence

Figure 22. Distribution of dislocation density for weak gradient dependence

Low Normal Pressure

Distocation

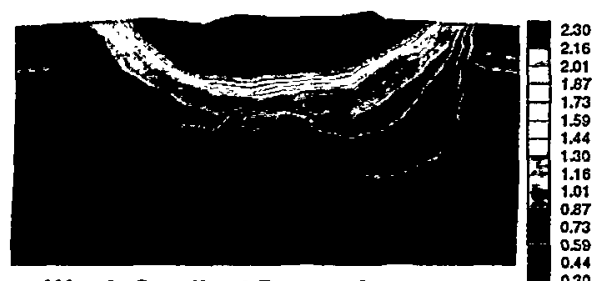

Weak Gradient Dependence

Figure 24. Distribution of dislocation density for weak gradient dependence and low stress.
High Normal Pressure

Dlestocation

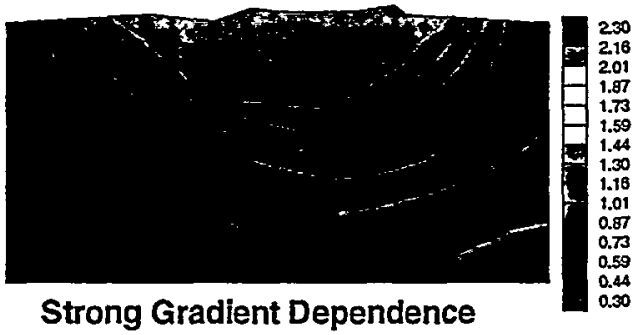

Figure 23. Distribution of dislocation density for strong gradient dependence.

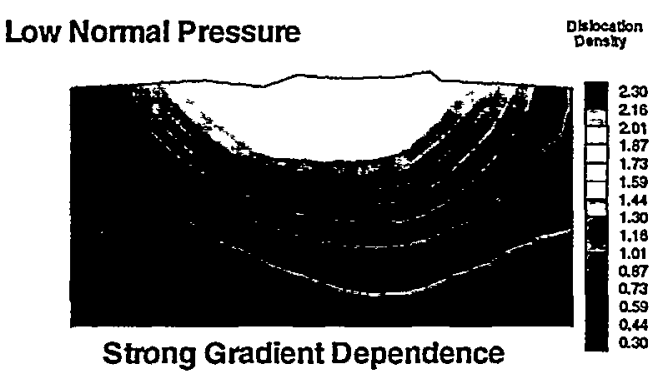

Figure 25. Distribution of dislocation density for strong gradient dependence and low stress.

\section{Length Scale Parameter Experiments}

In an effort to measure the physical length scale at which gradient effects become important, a novel experimental study was devised and conducted. In such types of experiments, there are two different approaches.

In the first, a single, very small-scale test is conducted which involves heterogeneous deformation. If the local behavior (uniform deformation) is known and modeled accurately enough, then any discrepancy between simulation with the local model and experiment can be attributed to gradient effects. This approach is not practical, since it would be very difficult to determine the anisotropic, large strain deformation behavior to the necessary precision.

In the second approach, the heterogeneous mechanical testing is performed at progressively smaller size scales. In this way, the magnitude of the strain 
gradients is increased by decreasing the size of identically proportioned specimens. One then anticipates that the mechanical response of the test specimens, normalized by the specimen dimensions in some manner, will be insensitive to specimen size until gradient effects influence the deformation mechanics. This approach requires that the local behavior be invariant between the differently sized specimens, but not that it be known or modeled to high precision. Because of this advantage, the second approach was pursued.

The greatest experimental challenge in this type of study is the fabrication of small-scale specimens that are properly proportioned and have identical microstructural states. Often the invariance of the local behavior can be evaluated either by slightly modifying the heterogeneous deformation test specimens or testing them in a different loading mode to produce uniform deformation. If these results depend on specimen size, then the local behavior of the specimens/material is improperly varying and any results from the associated heterogeneous tests must be corrected or interpreted with greater scrutiny.

Fleck et. al.[3] have conducted experiments in which the method of heterogeneous deformation was torsion of solid cylindrical wires. In that study, the normalized torque-rotation data indicated a change in behavior of polycrystalline copper wires for diameters of less than $15 \mu \mathrm{m}$. To verify invariance of the local behavior, the wires were pulled in uniform uniaxial tension. While there was some variation in the tension data, it was much less than the size effect seen in torsion, indicating a reasonably valid set of data for gradient effects. Greater discussion of these tests is given in Section 5.6.2.

The present experiments sought to study gradient effects in a similar manner but with a different specific configuration. A flat tensile specimen was used with a 6 by 30 array of holes as shown in Figure 26. The heterogeneities (holes) have a well-defined interfacial condition (tractionless). This contrasts alternate ways of producing gradients such as second phase particles or fibers for which the interfacial behavior would be an added unknown. The large number of unit cells within the specimen results in small-scale, high magnitude strain gradients while also producing a relatively high force response for experimental advantages. The two dimensional geometry simplifies specimen fabrication and metrology as well as meshing of the unit cell. 


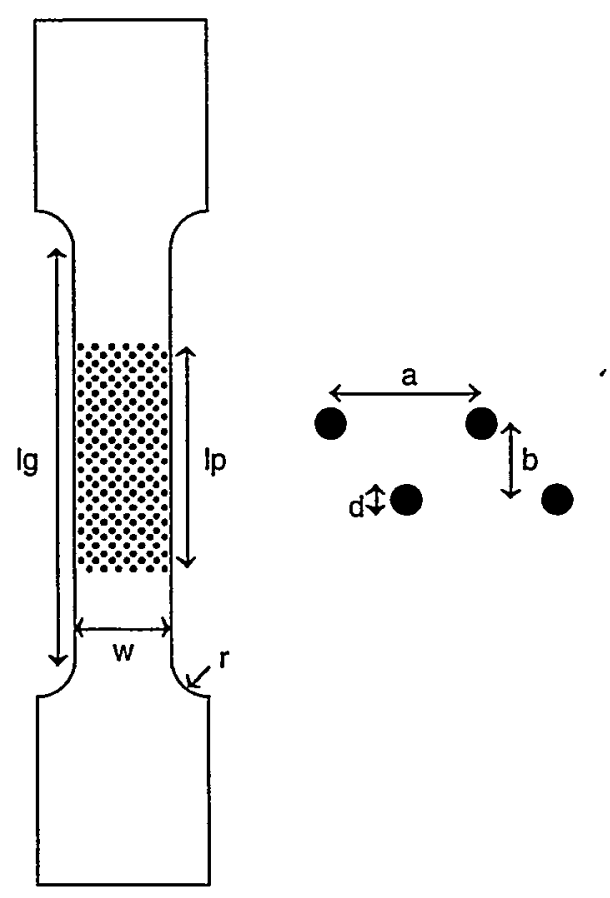

Figure 26. Perforated tensile specimen geometry with expanded view of hole configuration.

To produce the specimens, polycrystalline OFHC copper foil of $99.95 \%$ minimum purity was obtained from the Goodfellow Corporation ${ }^{1}$ in thicknesses of 0.025 , $0.076,0.254$ and $0.508 \mathrm{~mm}$. Resonetics, Inc. ${ }^{2}$ fabricated the identically proportioned specimens according to the specifications in Table 1. The specimen types are labeled " $A$ ", "B", " $C$ " and " $D$ " in order of increasing size. The two-dimensional hole configuration determines the magnitude of the strain gradient. Given this, it is then desired to make the specimens as large as possible for handling and test accuracy. The thickness was limited by the ability to produce holes with straight sides. The width was limited by concern of misalignment and wrinkling.

In the first step of the fabrication process, an etching house was sub-contracted to produce the dog-bone shaped outlines of the specimens shown in [30]. Next, Resonetics burned the holes with an Excimer laser, which uses a broad laser beam and patterned optical mask. The mask pattern contained a fraction of the total number of holes, requiring the mask to be repositioned to produce the complete hole array.

\footnotetext{
' Goodfellow Corporation, 800 Lancaster Ave., Berwyn, PA 19312, 800-821-2870.

${ }^{2}$ Resonetics, Inc., 4 Bud Way, \#21, Nashua, NH 03063, 800-468-7189.
} 
Table 1. Specifications for the perforated tensile specimen dimensions of Figure 26. The unit is millimeters.

\begin{tabular}{|c|c|c|c|c|c|c|c|c|}
\hline $\begin{array}{c}\text { Specimen } \\
\text { ID }\end{array}$ & Thickness & $\begin{array}{c}\text { Hole } \\
\text { Diameter }\end{array}$ & $\begin{array}{c}\text { Hole } \\
\text { Spacing }\end{array}$ & $\begin{array}{c}\text { Row } \\
\text { Spacing }\end{array}$ & $\begin{array}{c}\text { Perforated } \\
\text { Length }\end{array}$ & $\begin{array}{c}\text { Gage } \\
\text { Length }\end{array}$ & Radius & $\begin{array}{c}\text { Gage } \\
\text { Width }\end{array}$ \\
\hline & $\mathrm{t}$ & $\mathrm{d}=\mathrm{t} / 3$ & $\mathrm{a}=5 \mathrm{~d}$ & $\mathrm{~b}=\mathrm{a} / 2$ & $\mathrm{l}=29 \mathrm{~b}$ & $\mathrm{Ig}=\mathrm{lp}+2 \mathrm{w}$ & $\mathrm{r}=3 \mathrm{a}$ & $\mathrm{w}=6.5 \mathrm{a}$ \\
\hline $\mathrm{A}$ & 0.0254 & 0.0085 & 0.0423 & 0.0212 & 0.614 & 1.16 & 0.127 & 0.275 \\
\hline $\mathrm{B}$ & 0.076 & 0.025 & 0.127 & 0.064 & 1.84 & 3.49 & 0.381 & 0.826 \\
\hline $\mathrm{C}$ & 0.254 & 0.085 & 0.423 & 0.212 & 6.14 & 11.6 & 1.27 & 2.75 \\
\hline $\mathrm{D}$ & 0.508 & 0.169 & 0.847 & 0.423 & 12.3 & 23.3 & 2.54 & 5.50 \\
\hline
\end{tabular}

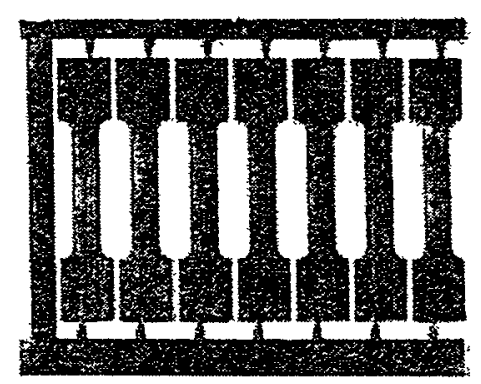

Figure 27. Specimen blanks of the "C" size produced by chemical etching, shown at approximately a 1:1 scale.

The actual specimen geometry of the smallest two sizes can be seen in Figure 28. Measured specimen dimensions are given in Table 2 for all but the " $D$ " type specimens. The fabricated hole patterns agree well with the specifications for all of the specimens except for the "A" size, which was understood a priori to push the resolution of the Excimer laser. The hole diameters were generally in error by about $0.003 \mu \mathrm{m}$ which was acceptable except for the "A" specimens. An attempt was made to put fiducial lines on the specimens, but they were not well defined enough to be of use. Resonetics estimated a hole taper angle of 7 degrees, but measurements of hole diameters on the front and back sides were essentially the same, and the taper angle was estimated to be less than 1 degree. 

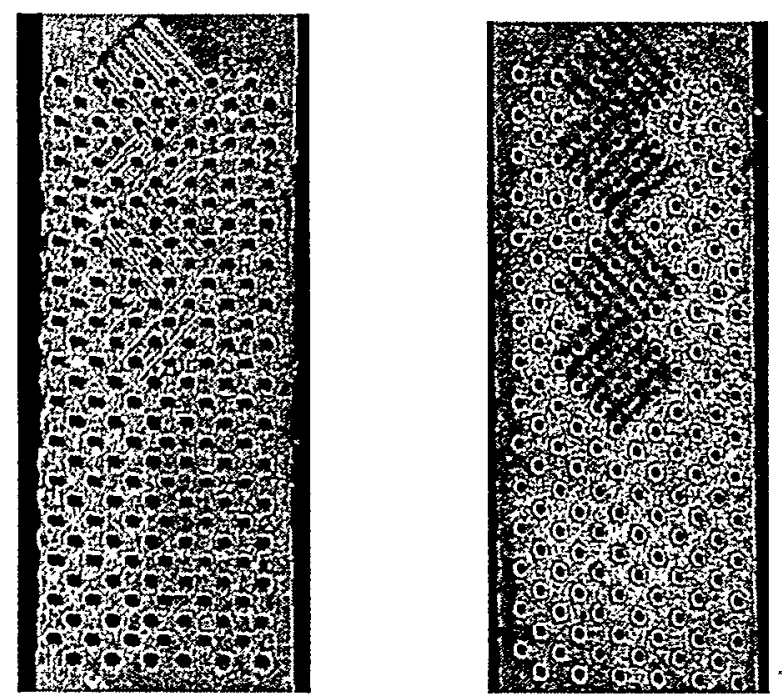

Figure 28. Geometry of an "A" specimen (left) with 8-10 $\mu \mathrm{m}$ holes and a "B" specimen (right) with $28 \mu \mathrm{m}$ holes.

Table 2. Measured dimensions of fabricated specimens " $A$ " - " $C$ ".

\begin{tabular}{|c|c|c|c|}
\hline Specimen ID & Thickness, $t$ & Hole Diameter, $\mathrm{d}$ & Hole Spacing, a \\
\hline A & 0.0259 & 0.0107 & 0.041 \\
\hline B & 0.0775 & 0.028 & 0.130 \\
\hline C & 0.245 & 0.081 & 0.427 \\
\hline
\end{tabular}

Close-up views of the " $A$ " and " $B$ " specimens are shown in Figure 29. This emphasizes the difference in quality of the hole pattern between the two smallest sizes. The irregularity in the hole spacing of " $A$ " was caused by misalignment of the optical mask when it was repositioned. Figure 28 shows, however, that overall the hole spacing is reasonably acceptable.
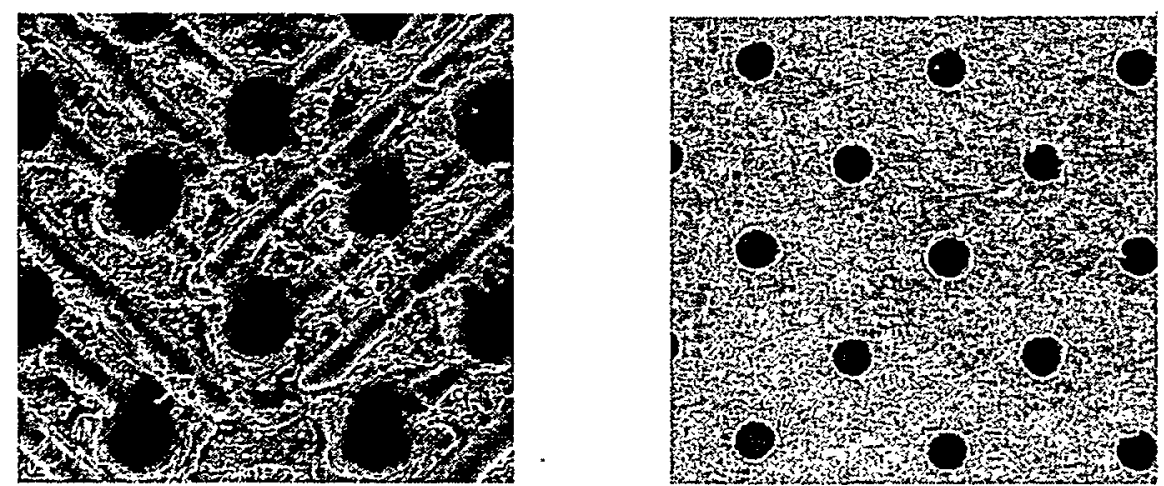

Figure 29. Close up views of the front side of specimen " $A$ " (left) and back side of specimen " $B$ " (right).

Alternate fabrication methods were considered which would need to have both fine resolution for the small specimens and remove material fast enough for the 
large specimens. Conventional drilling and EDM both have size limits in the 0.08 to $0.12 \mathrm{~mm}$ range. Focused beam laser cutting ( $\mathrm{CO} 2$ and $Y A G$ ) has a similar size limitation and may not produce round holes. Focused ion beam milling has high precision but low material removal rates - cutting of a single hole for the smallest specimen would take roughly $\$ 1,000$ of beam time. Wet chemical and plasma etching remove material as a penetrating hemisphere, which is not appropriate for this particular specimen geometry. The LIGA method, for build up rather than removal of material, is a possible alternative for fabrication of polycrystalline specimens, but this method was not practical for this particular project.

After the specimens were fabricated and received from Resonetics, they were vacuum annealed at 600 degrees Celsius for 4 hours. The microstructure and heat affected zone (HAZ) from the hole burning is shown in Figure 30 for specimen "C". This HAZ was the largest, relative to the hole diameter, of any of the specimens examined and had a thickness that was about $15 \%$ of the hole diameter.

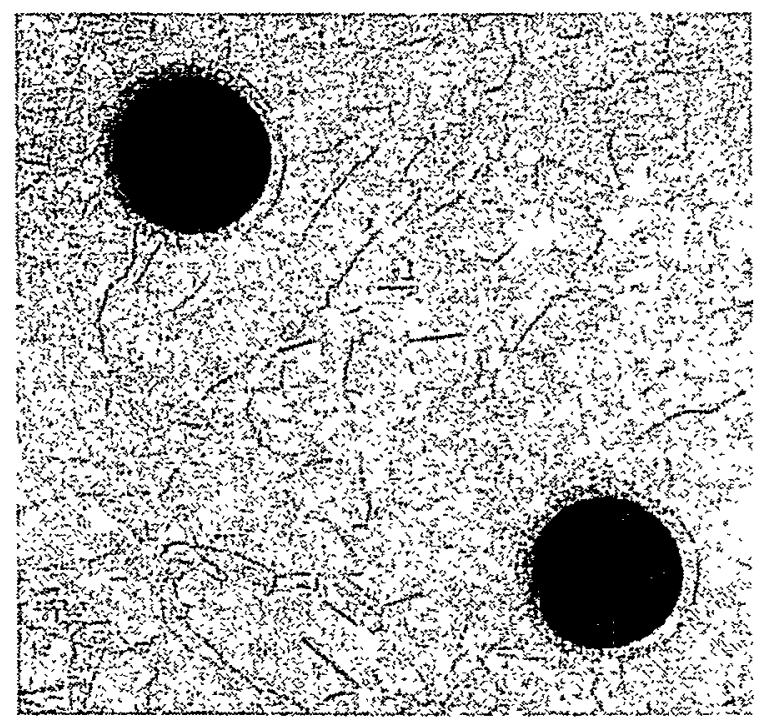

Figure 30. Microstructure and heat affected zone for a type "C" specimen, the largest $\mathrm{HAZ}$ observed.

The smallest three specimen sizes ("A" through "C") were tested on a custom load frame designed for relatively small specimens. For a description of this system, see Schmale et al..$^{31}$ The " $D$ " specimens were too large for this system and were tested on a conventional Instron servo-mechanical machine. Micrographs of the original and deformed unit cells are given in Figure 31. In addition to the perforated specimens, tests were conducted on solid specimens (without holes) to evaluate invariance of the local behavior. 

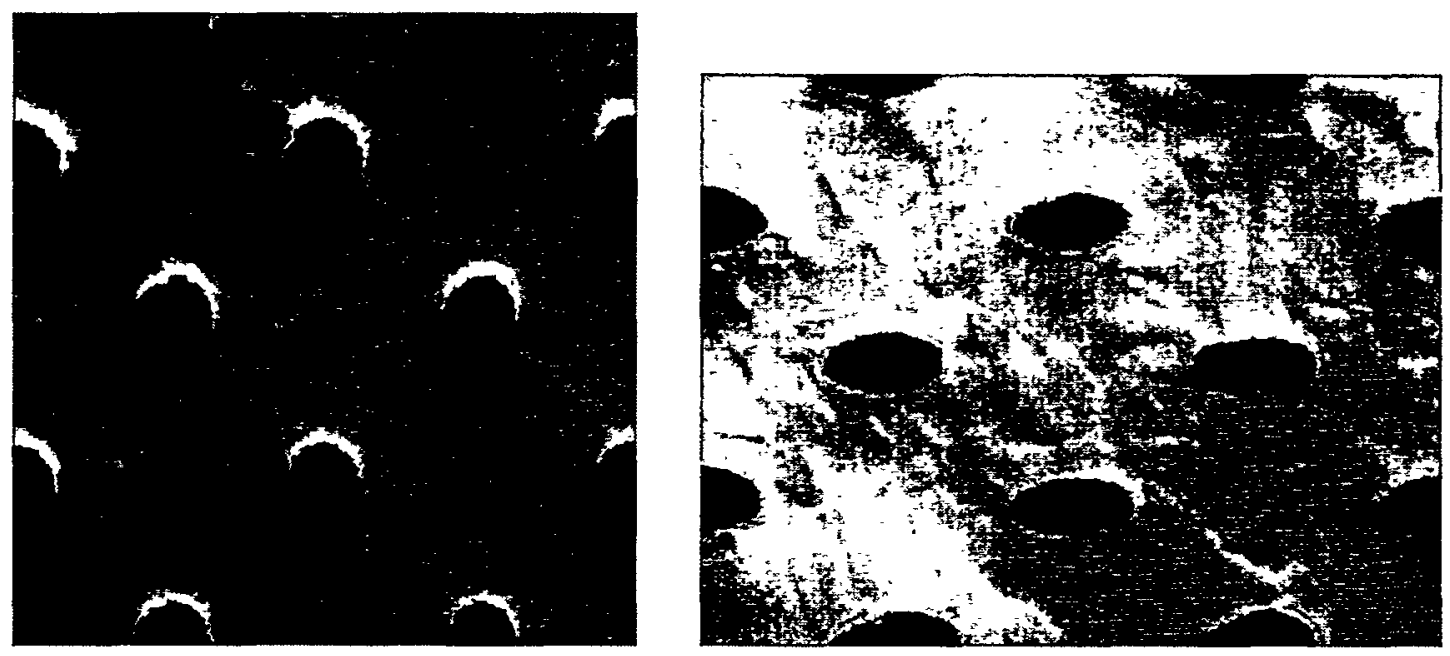

Figure 31. Undeformed and deformed unit cell geometry for a " $\mathrm{B}$ " specimen.

Because fine scale extensometry was not available to measure the straining in the perforated gage section, the specimen deformation was based on displacement measurements made between the grips. This introduces complications due to the deformation of the solid portion of the gage section and fillets but not from machine compliance. Since the measurements are selfsimilar, however, comparisons can still be made to examine the size scale at which gradients become influential. Time stamped video recordings were made through a microscope in case it became important to have measurements of just the perforated gage section deformation. The time indicator, shown in Figure 32, was synchronized with the load and displacement data.

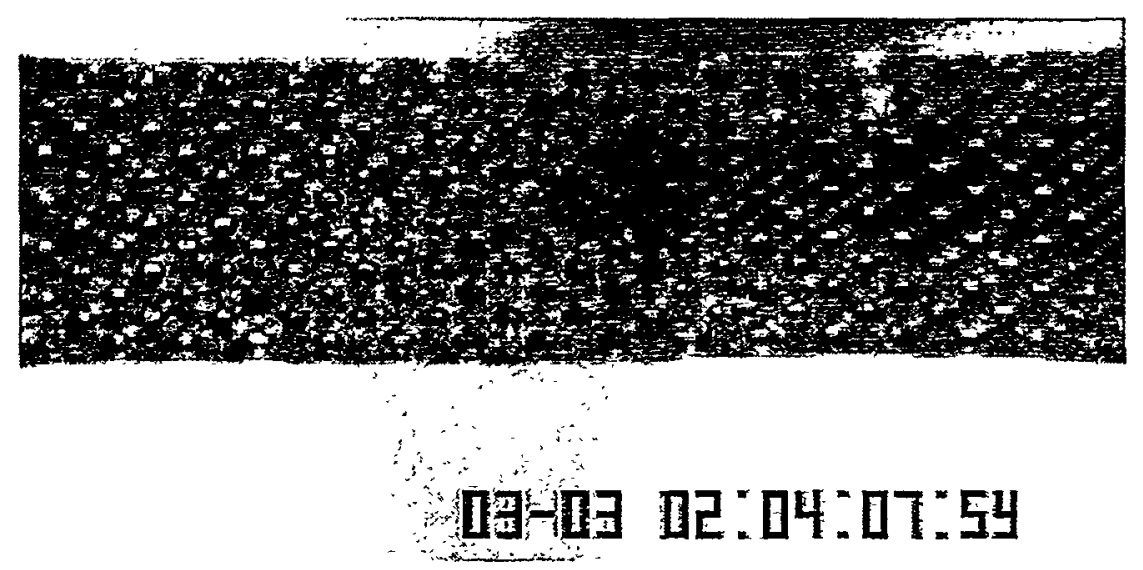

Figure 32. Video recording of deformation with synchronized time indicator (March $3^{\text {rd }}, 2^{\text {nd }}$ test, 4 minutes 7 seconds into test) for a "C" specimen.

Force and displacement data are graphed in Figure 33 in which repeatability is seen to be reasonably good for both the solid and perforated " $C$ " specimens. The plastic flow behavior for the solid and perforated specimens is very similar 
initially; at larger strains the holes soften the specimen response. One might expect that the perforations would significantly reduce the yield strength, but the holes comprise only $6 \%$ of the volume. In addition, it is likely that the holes increase the stress triaxiality or hydrostatic tension in the copper, as in a notched tensile specimen. This results in a larger axial traction for a given deviatoric stress, which compensates for the loss of material.

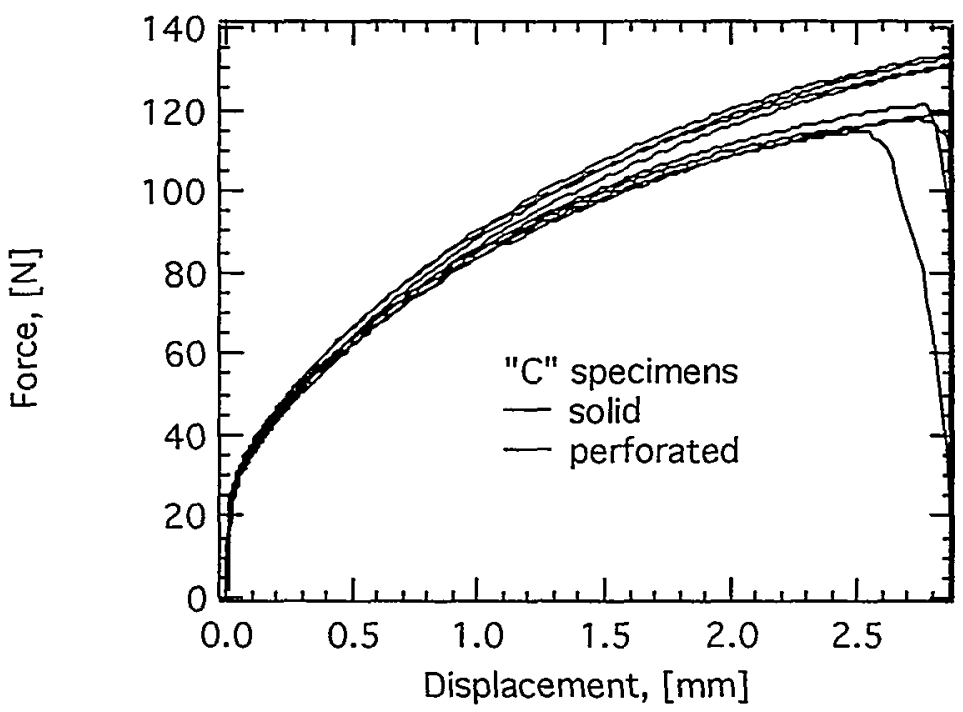

Figure 33. Force and displacement data for both the solid (uniform straining) and perforated (strain gradients) " $\mathrm{C}$ " specimens.

The force versus displacement plot for the "A" solid specimens is given in Figure 34. The results are seen to lie in two bands. Similar banding was also observed in the perforated "A" specimens. At first, the cause was suspected to be specimen geometry, but subsequent evaluation indicated this was not the case. Another possible cause is misalignment and wrinkling of the specimens, since their small size made them difficult to handle precisely. While this was not ruled out, it is unlikely that the data would fall into two bands, but rather be more uniformly distributed. Variation in the initial slope may be due to this misalignment/wrinkling. A more plausible cause for the banding is the number of grains through the specimen thickness.

Microstructures for the three smallest specimen sizes are shown in Figure 35. From this, the number of grains through the thickness can be examined. The "A" specimen has sections with only one grain while other portions have two or more grains. This may be the cause of the two types of response in Figure 34. When a specimen has a substantial portion with only one cross-thickness grain, there is negligible intergranular constraint on the slip systems and the response is softer. 
This effect is undesirable in this study, since it alters the local behavior and makes it more difficult to estimate the effects of strain gradients.

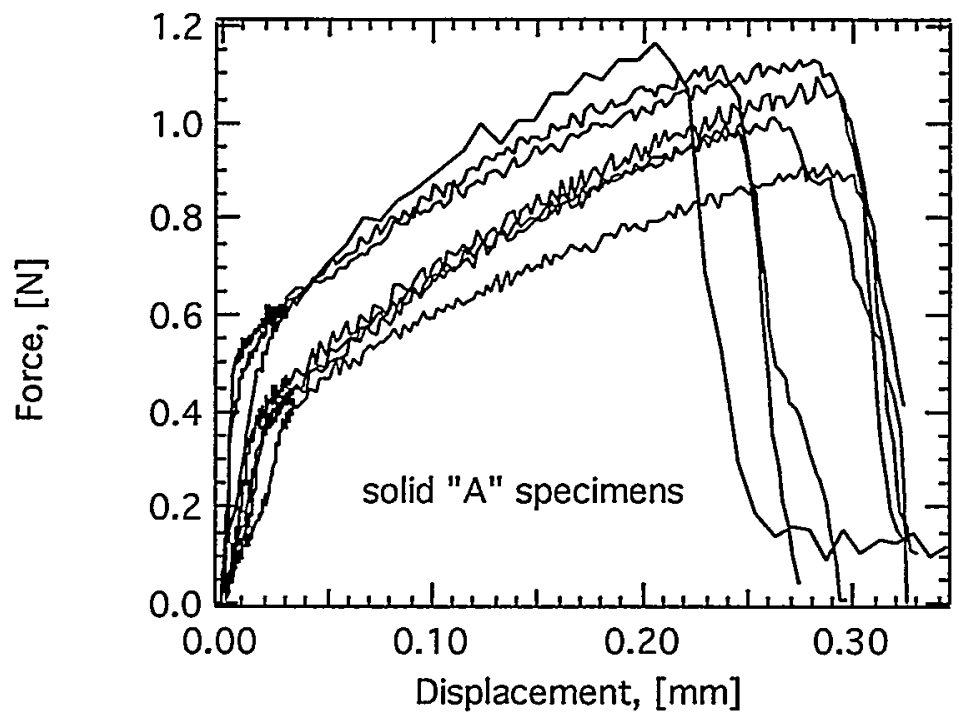

Figure 34. Force and displacement data for solid "A" specimens. The data lie in two bands.
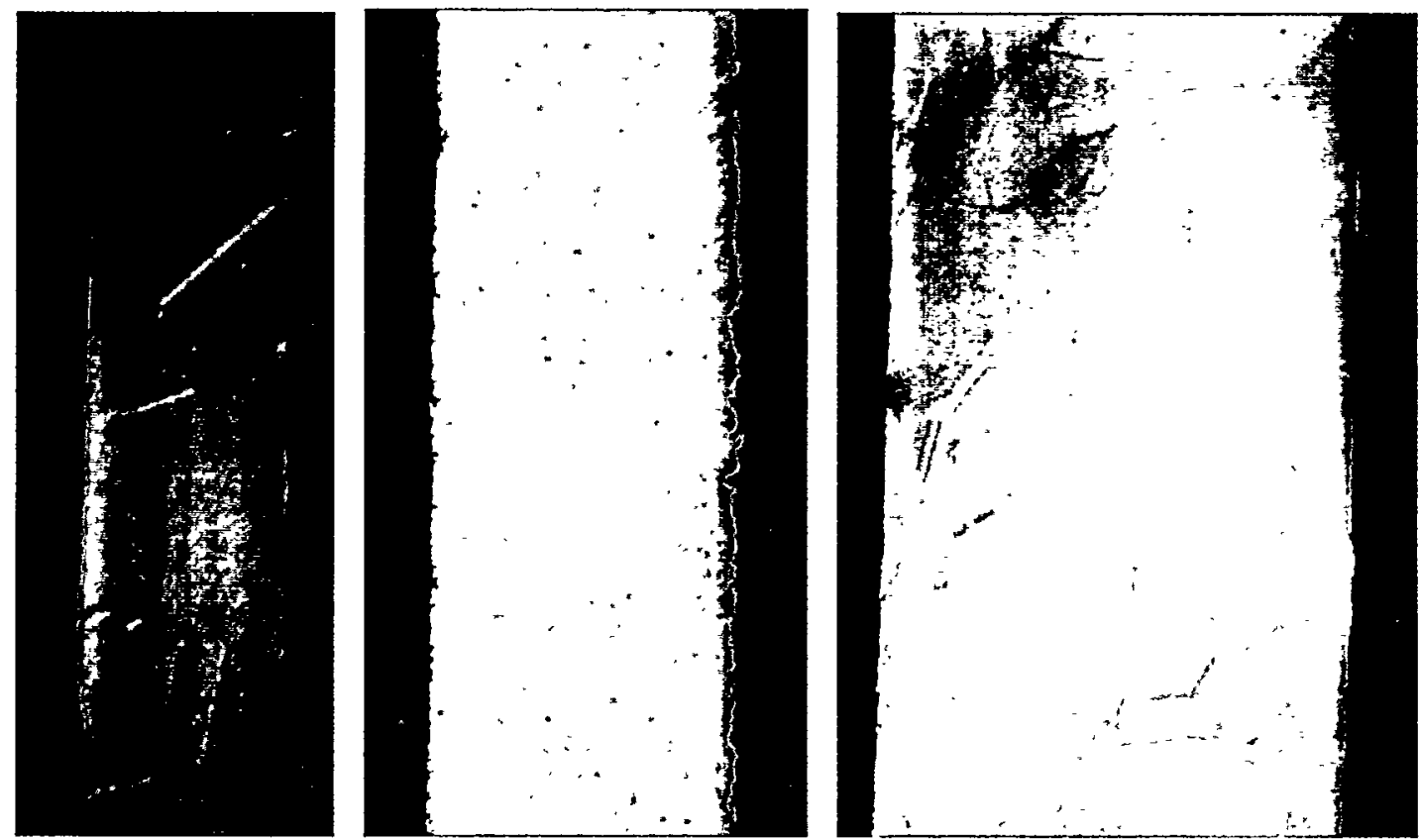

Figure 35. Micrographs through the thickness dimension for (left to right) " $A$ ", " $B$ " and " $C$ ". The magnification is different for each micrograph, but can be determined from the thicknesses in Table 2. 
The force data was normalized by the cross sectional area (initially equal to $t^{*} w$ ) to produce a measure of stress. The evolution of the cross sectional area was based on assuming that the deformation is isochoric,

$$
\text { area }=\frac{\mathrm{t} * \mathrm{w} * \mathrm{lg}}{\mathrm{lg}+\mathrm{u}}
$$

where " $\mathrm{u}$ " is the displacement data. This is not a rigorously justified assumption, but is adequate for the purpose of measuring relative differences in scaled, selfsimilar tests. Likewise, a nominal strain was based on the total length of the gage section,

$$
e=\ln \left(\frac{\lg +u}{\lg }\right) .
$$

Multiple trials were conducted and the data were averaged. In the case of the "A" specimen size, data from both bands were included in the average. The largest specimens had to be tested on a different system and the method of displacement measurement was slightly different. The results are shown in Figure 36. The largest three perforated specimen results nearly overlap. The middle two solid specimens also scale consistently, but the solid " $\mathrm{D}$ " does not. It is unknown what caused this discrepancy, but it could be due to the different method of displacement measurement. Both the solid and perforated "A" stressstrain curves are lower than the other sizes. While this could be interpreted as a length scale effect, it is most likely caused by the reduced intergranular constraint due to having only one grain through the thickness as shown in Figure 35. Since the solid specimen behavior is different, the uniform or local behavior has changed, which requires further interpretation to infer the effect of gradients.

One approach to correct for changes in local behavior is to normalize the perforated specimen stress by the solid specimen stress. Figure 37 gives this ratio for the stresses at a strain of 0.15 . No length scale effects associated with gradients can be discerned, especially when considering the scatter in the " $\mathrm{A}$ " specimen data of Figure 34. Nanoindentation tests were conducted on the deformed specimens, but the results were inconclusive. While the present experimental results do not uniquely determine a gradient length scale parameter, they can be used to estimate an upper bound. 


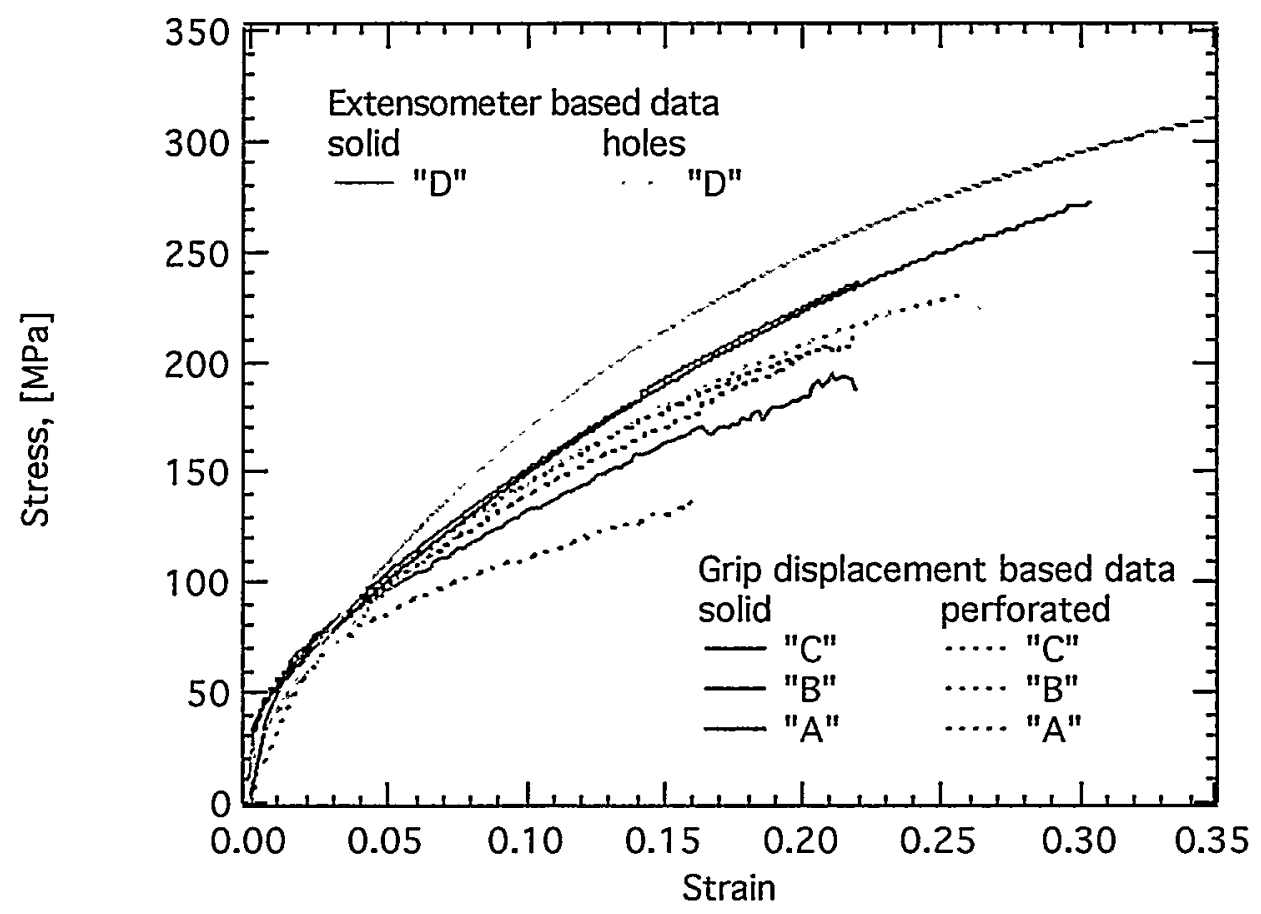

Figure 36. Averaged stress - strain response for specimens of all sizes.

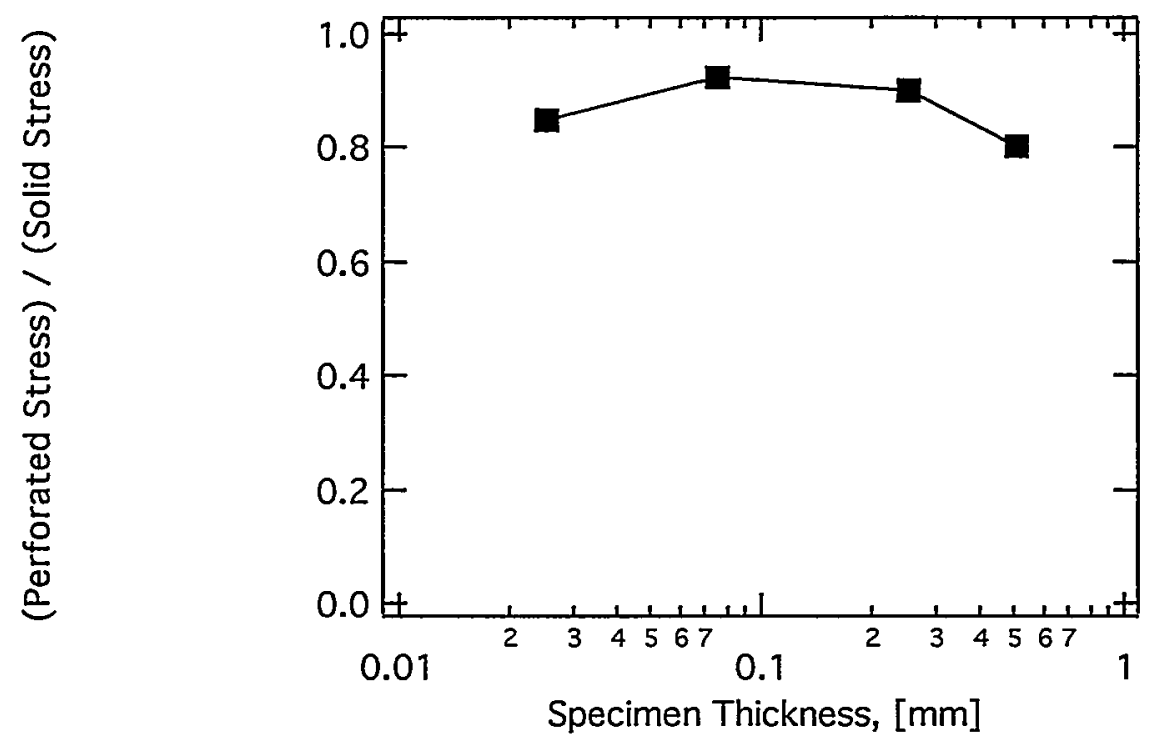

Figure 37. Ratio of the flow stress for the perforated specimens over the flow stress for the solid specimens for the data in Figure 36 at nominal strain of 0.15 .

In addition to examining the effect which gradients have on the pre-localization strain hardening, the perforated specimen configuration also had the potential to 
study post-localization effects such as shear band width. The anticipated localization mode was a 45-degree shear band. Due to the free edge effects, localization initiated there and propagated inward, similar to a mode I crack. In some cases, this tearing transitioned into a 45-degree shear band as shown in Figure 38. However, because the boundary conditions are not well defined for the shear band unit cell, experimental probing of such shear bands would have limited benefit.

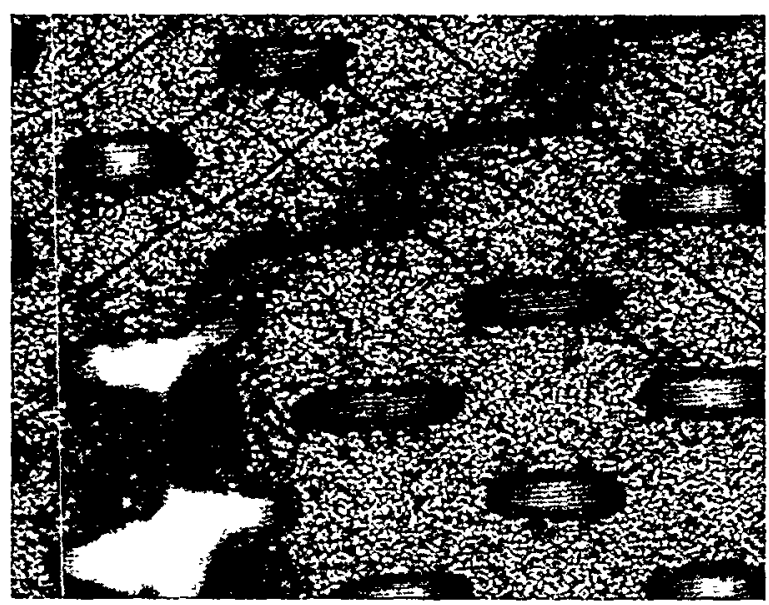

Figure 38. Transition of localization from a mode I "crack" type tearing to a shear band ("D" size specimen).

One issue that arose in this study is that even the prescription of the desired microstructure becomes problematic, especially for polycrystalline materials. Should the grain size or number of grains be kept constant as the specimen size is varied? One approach is to choose a grain size that gives a constant local behavior, i.e. under relatively uniform deformation. If there were large numbers of grains through the specimen dimensions, then a constant grain size would seem to be desirable. As the number of grains across key regions approaches unity, intergranular constraint is reduced, and the local behavior is softened. A smaller grain size might then harden the local behavior back to the previous level. Such an approach has limitations, however, and could lead to results that depend on the specific specimen geometry.

Since the influence of non-local effects depends on the interplay between strain gradients and heterogeneities in the microstructure, the material can not simply be made homogeneous to eliminate the above complications. If grain interaction is an important aspect of non-local behavior, then using single crystals in scaled tests to give invariant local behavior would not result in the response of interest when gradients are introduced. Because of these complexities in precisely controlling the material, micromechanical simulation "experiments" would seem to be advantageous in evaluating the interaction of strain gradients and microstructural heterogeneities. 


\section{Impact and Recommendations}

The use of non-local or gradient models in the description of the inelastic response of metals is an active area of research. These models are used in cases of post strain localization or damage propagation to allow solution of boundary value problems in the post-bifurcation regime and eliminate the mesh dependency of the solution. This aspect is becoming more important as more information is demanded of the finite element solution. We have extended classical internal state variable models in a thermodynamically consistent manner and proposed a method of implementation into finite element codes. The importance of the length scale introduced by the spatial gradient on post bifurcation behavior of strain localization and damage propagation has been investigated, and shown to result in reasonable solutions in this regime. This model has recently been used by Paul Maudlin of LANL to predict the deformation of a shape charge. This was an essential part of the solution, in that localization and hence mesh dependency of the solution occurred in the early stages of deformation (private communication). The importance of the gradient terms in the solution of very small length scale problems was also investigated and shown to result in reasonably accurate solutions in cases outside the range of local continuum mechanics.

The use of these types of models has a restricted but important use. It would certainly be overkill to use these types of models for problems of a large enough length scale or when only information prior to bifurcation is required. However, in cases where post bifurcation analyses are important and in cases where the actual deformation can not be described by local continuum models, it will be important to have efficient implementation of non-local or gradient models in SNL finite element codes. 


\section{Appendix}

Hardening equations.

Hart Model:

$$
H_{\kappa}=c_{0} \kappa\left(\frac{G}{\kappa}\right)^{m^{\prime}}\left(\frac{\tau^{\prime} p}{\kappa}\right)^{n} D^{\prime}
$$

BCJ Model using hardness:

$$
H_{K}=H_{0} D^{\prime}-\left(R_{S}-R_{D}\right) D^{\prime} \rho
$$

BCJ Model using dislocation density:

$$
H_{\kappa}=\left(\frac{H_{0}}{\sqrt{\rho}}-R_{D}\right) D^{\prime} \rho
$$

Lee-Dawson Model:

$$
\mathrm{H}_{\Phi}=\Phi_{0} \exp \left(c_{2} \frac{\sigma_{\mathrm{m}}}{\kappa}\right) \mathrm{D}^{\prime}\left(\frac{\Phi}{1-\Phi}\right)
$$

Table 1. Material Parameters for the Simplified Hart's Model for Aluminum.

\begin{tabular}{|c|c|c|c|}
\hline $\begin{array}{c}a_{0} \\
\left(\mathrm{~s}^{-1}\right)\end{array}$ & $\begin{array}{c}f_{0} \\
\left(\mathrm{~s}^{-1}\right)\end{array}$ & $\begin{array}{c}\mathrm{G} \\
(\mathrm{GPa})\end{array}$ & $\begin{array}{c}\kappa_{0} \\
(\mathrm{MPa})\end{array}$ \\
\hline $9.64 \times 10^{52}$ & $2.12 \times 10^{19}$ & 24. & 64.0 \\
\hline $\begin{array}{c}\mathrm{Q}_{0} / \mathrm{R} \\
(\mathrm{K})\end{array}$ & $\begin{array}{c}\mathrm{Q}^{1} / \mathrm{R} \\
(\mathrm{K})\end{array}$ & $\mathrm{c}_{0}$ & $\tau$ \\
\hline $1.45 \times 10^{4}$ & $1.45 \times 10^{4}$ & $6.19 \times 10^{-9}$ & 0.15 \\
\hline $\mathrm{M}$ & $m$ & $n$ & $m^{\prime}$ \\
\hline 7.8 & 5.0 & 4.5 & 3.5 \\
\hline
\end{tabular}


Table 2. Material Parameters for the Damage Model for Aluminum.

\begin{tabular}{|c|c|c|c|c|}
\hline$c_{1}$ & $c_{2}$ & $D_{r}$ & $\theta_{r}$ & $\kappa_{r}$ \\
$(-)$ & $(-)$ & $\left(\mathrm{s}^{-1}\right)$ & $(\mathrm{K})$ & $(\mathrm{MPa})$ \\
\hline 1.6 & 24.0 & 1.0 & 373.0 & 64.0 \\
\hline
\end{tabular}

Table 3. Material Parameters for the Dislocation Density Evolution Model.

\begin{tabular}{|c|c|c|c|}
\hline $\begin{array}{c}\text { Material } \\
-\end{array}$ & $\begin{array}{c}H_{o} \\
\left(\mathrm{~m}^{-1}\right)\end{array}$ & $\begin{array}{c}R_{D} \\
-\end{array}$ & $\begin{array}{c}P_{i} \\
\left(\mathrm{~m}^{-2}\right)\end{array}$ \\
\hline Copper & 3208 & 9.13 & $0.22 \times 10^{16}$ \\
\hline Stainless & 7118 & 5.265 & $4.29 \times 10^{16}$ \\
\hline
\end{tabular}

${ }^{1}$ Aifantis EC, "From Micro-Plasticity To Macro-Plasticity: The Scale-Invariance Approach", Journal Of Engineering Materials And Technology-Transactions Of The ASME, v. 117 (\#4) pp. 352-355 Oct 1995

${ }^{2}$ Dillon O. W., and J. Kratochvil, "A Strain Gradient Theory of Plasticity", Int. J. Solids and Struct., v. 6, pp.1533-1566, 1970

${ }^{3}$ Fleck N.A., Muller G.M., Ashby M.F., Hutchinson J.W., "Strain Gradient

Plasticity: Theory And Experiment", Acta Metallurgica Et Materialia, v. 42 (\#2) pp. 475-487 FEB 1994

${ }^{4}$ Nix W.D., Gao H.J., "Indentation Size Effects In Crystalline Materials: A Law For Strain Gradient Plasticity", Journal Of The Mechanics And Physics Of Solids, v. 46 (\#3) pp. 411-425 MAR 1998

${ }^{5}$ Coleman, B.D. and M. E. Gurtin, Thermodynamics with Internal State Variables", J. Chem. Phys., v.47 (\#2), pp497, Jul 1967

${ }^{6}$ Teodosiu, C, "Thermodynamics of Inelastic Deformation", Bull. Acad. Polon. Sci., v.15, p103, 1967

7J. Kratochvil and O. W. Dillon, "Therrmodynamics of Crystalline Elastic-ViscoPlastic Materials", J. Appl. Phys., v. 41 (\#4), Mar 1970

${ }^{8}$ Kelly, J.M. and P.P. Gillis,

9 Perzyna, P., "The Constitutive Equations for Rate Sensitive Plastic Materials", Quart. Appl. Math., v. 20,p.321, 1963 
${ }^{10}$ Brown S.B., K.H. Kim and L Anand, "An Internal Variable Constitutive Model For Hot-Working Of Metals", International Journal Of Plasticity, v. 5(\#2) pp. 951301989

${ }^{11}$ Bammann, D.J., "An Internal Variable Model of Visco-Plasticity", Int. J. Eng. Sci.,v.22, pp.1041-1053, 1984

${ }^{12}$ Mcdowell, D.L., "A Bounding Surface Theory For Cyclic Thermoplasticity", Journal Of Engineering Materials And Technology-Transactions Of The ASME, v. 114 (\#3) pp. 297-303 JUL 1992

${ }^{13}$ Bilby, B.A., R. Bullough and E. Smith, "Continuous Distributions of

Dislocations: A New Application of the Methods of Non-Riemannian Geometry", Proc. Royal Soc. London, A236, PP481-505, 1956

${ }^{14}$ Lee, E.H. and D.T. Liu, Finite Strain Elastic-Plastic Theory with Application to Plane-Wave Analysis", J. Appl. Phys., v. 38 (\#1), JAN 1967

${ }^{15}$ Ramaswamy, S and N Aravas, "Finite-Element Implementation Of Gradient Plasticity Models: Part I : Gradient-Dependent Yield Functions", Computer Methods In Applied SEP 21, Mechanics And Engineering, v. 163 (\#1-4) pp. 1132, 1998

${ }^{16}$ Tvergaard, $V$ and $A$ Needleman, "Effects Of Nonlocal Damage In Porous Plastic Solids", International Journal Of Solids And Structures, v. 32 (\#8-9) pp. 1063-1077, APR-MAY 1995

${ }^{17}$ Holt, J, "Modeling Dislocation Substructure", J. Appl. Phys., v.48, p42, 1967

${ }^{18}$ Pilecki, S. "Introduction to the Diffusional Theory of Metal Fatigue", Third International Conference on Fracture, Munich, v1, pp.241-245, 1973

${ }^{19}$ Nunziato, Jwand S.C. Cowin, "Non-Linear Theory Of Elastic-Materials With Voids", Archive For Rational Mechanics And Analysis, v. 72 (\#2) pp. 175-201 1979

${ }^{20}$ Bammann, D.J. and E.C. Aifantis, "On A Proposal For A Continuum With Microstructure", Acta Mechanica, v. 45(\#1-2) pp. 91-121 1982

${ }^{21}$ Walgraef, D and E.C. Aifantis, "Dislocation Patterning In Fatigued Metals As A Result Of Dynamical Instabilities", Journal Of Applied Physics , v. 58(\#2) pp. 6886911985

${ }^{22}$ Fried, E and M.E. Gurtin, "Continuum Theory of Thermally Induced Phase

Transitions Based on an Order Parameter", Physica D, v. 68, pp. 326-343, 1993

${ }^{23}$ Grach, G, M Lusk and D.J. Bammann, "Dislocation Plasticty Using a Modulated Energy Function", submitted to J. Mech. Phys Solids

${ }^{24}$ Gurtin, M.E., "A strain Gradient Theory of Plasticity", to appear plastic gradient 
${ }^{25}$ Wilsdorf, D.K. and FRN Nabarro, "Nucleation Of Small-Angle Boundaries", Scripta Materialia, v. 35(\#11) pp. 1331-1333 DEC 1, 1996

${ }^{26}$ Mecking, H. and U.F. Kocks,"Hardening in Simple Glide", Acta Mettall.,v29, p1865, 1981

${ }^{27}$ Dawson P.R. and E.G. Thompson, "Finite-Element Analysis Of Steady-State Elasto-Visco-Plastic Flow By Initial Stress-Rate Method", International Journal For Numerical Methods In Engineering, v. 12 (\#1) pp. 47-57, 1978

${ }^{28}$ Dawson, P.R. and P.S. Follansbee, "The Variable Threshold Rod Experiment: A Comparison Of Measured And Computed Deformations", Journal Of Engineering Materials And Technology-Transactions Of The ASME, v. 115 (\#4) pp. 446-454 OCT 1993

${ }^{29}$ Dawson, P, YS Lee and A Kumar," Void Growth In Drawing And Extension Of Ductile Metals", Journal Of Materials Processing Technology, v. 32 (\#1-2) pp. 119-134 JUL 1992

${ }^{30}$ Hughes, D.A., D.B. Dawson, J.S. Korellis and L.I. Weingarten, "Near-Surface Microstructures Developing Under Large Sliding Loads", Journal Of Materials Engineering And Performance, v. 3(\#4) pp. 459-475, AUG 1994

${ }^{31}$ Schmale, D.T., R.J. Bourcier and T.E. Buchheit, "Description of a MicroMechanical Testing System," Sandia Report, SAND97-1608, 1997. 


\section{Distribution:}

3 Prof. Paul R. Dawson

Cornell University

196 Rhodes Hall, Hoy Road

Ithaca, NY 14853

$1 \quad$ MS 0149

C. E. Meyers, 4000

1 MS 9042

E. P. Chen, 8726

1 MS 9042

W. A. Kawahara, 8725

1 MS 9405 M. T. Dyer, 8700

1 MS 9405 C. M. Hartwig, 8700

1 MS 9405 P. E. Nielan, 8920

1 MS 9405 D. A. Hughes, 8726

1 MS 9405 N. R. Moody, 8725

25 MS $9405 \quad$ D. J. Bammann, 8726

3 MS 9018 Central Technical Files, 8940-2

1 MS 0899 Technical Library, 4916

1 MS 9021 Technical Communications Department, 8528/

Technical Library, MS 0899, 4916

1 MS 9021 Technical Communications Department, 8528 For DOE/OSTI

1 MS 0188 D. Chavez, LDRD Office, 4001 